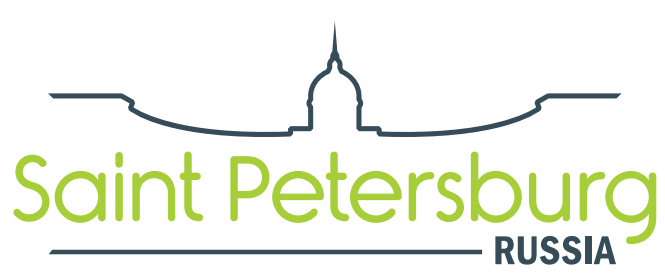

B30

\title{
Dynamic Interactions between Matrix and Fracture in Miscible Solvent Flooding of Fractured Reservoirs
}

\author{
A. Ameri Ghasrodashti* (Delft University of Technology), R. Farajzadeh \\ (TU Delft and Shell Global Solutions International), M. Verlaan (Shell \\ Canada Limited), V.S. Suicmez (Brunei Shell Petroleum) \& H. Bruining \\ (Delft University of Technology)
}

\section{SUMMARY}

Miscible solvent injection has received increasing attention in recent years as an efficient method to improve oil recovery from fractured reservoirs. Due to the large permeability difference between fracture and matrix, the success of this method depends to large extent on the degree of enhancement of the mass exchange rate between the solvent flowing through the fracture and the oil residing in the matrix.

A series of experiments have been conducted to investigate the mass transfer rate between the fracture and the matrix. Different scenarios have been considered to examine the effect of flow rate, matrix permeability, fracture aperture, and oil properties. To this end a porous medium (fully saturated with oil) is placed in a vertical core holder that can be used in a CT scanner, to simulate the matrix. A small slit between the porous medium and the core holder simulates the fracture. The interaction between the matrix and fracture is visualized for solvent flooding by means of CT-Scanning, which can be used to validate theories of enhanced transfer in fractured media. The experimental data are compared with a simulation model that takes diffusive, gravitational and convective forces into account. 


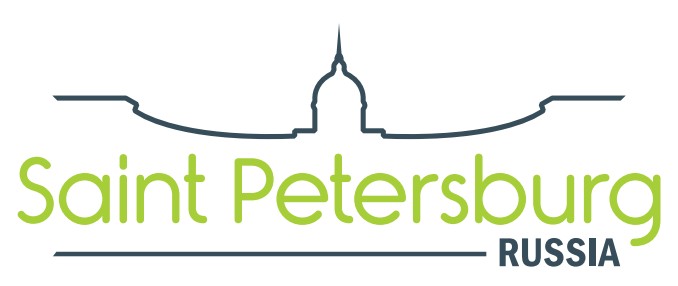

\section{Introduction}

A large part (20-30\%) of the oil resources is present in fractured reservoirs (Firoozabadi 2000). However, a large fraction of these naturally fractured reservoirs (NFR) do not show high production performance as most of the oil resides in matrix blocks from which it is difficult to recover, unless enhanced oil recovery techniques are applied. Miscible displacement processes have been developed as successful method to improve oil recovery from NFRs. The success of miscible displacement process in NFRs largely depends on the degree of mass exchange between the solvent in the fracture and the oil in the matrix (Er and Babadagli 2010, Kahrobaei et al. 2012, Trivedi and Babadagli 2008, Uleberg and Høier 2002, Verlaan and Boerrigter 2006, Yanze and Clemens 2012). Generally, four driving forces, i.e., viscous forces, gravity forces, capillary forces and diffusion effects influence the fluid transfer between matrix and fracture. When the injected fluid is fully miscible with the oil in the matrix, there is no capillary-driven cross-flow between matrix and fracture. Moreover, for heterogeneous layered fractured reservoirs, which are even more adversely affected by capillary effects, miscible flooding adds the advantage of interfacial tension elimination and thus enhancing the drainage rate and microscopic sweep efficiency. On the other hand, due to the existence of highly conductive and interconnected fracture networks, viscous forces are usually negligible compared to gravity forces (Salimi and Bruining 2010). Consequently, gravity (convection) drive and diffusion/dispersion drive are considered to be the dominant mechanisms responsible for the miscible recovery from fractured reservoirs. A major concern for miscible fluid injection in fractured oil reservoirs is the early breakthrough and production of large quantities of the injected fluid. Therefore, understanding the matrix-fracture communications is essential in order to optimize the recovery performance during miscible flooding. Experimental results (Firoozabadi and Markeset 1994, Trivedi and Babadagli 2008) and numerical simulations (Hoteit and Firoozabadi 2009, Yanze and Clemens 2012) reveal that for viscous oils diffusion and mixing in the matrix and fracture (Verlaan and Boerrigter 2006) are the most important factors governing the efficiency of miscible flooding in fractured reservoirs. In an experimental study, Trivedi and Babadagli (2008) examined the effect of oil-phase properties, water saturation, and injection rate on the oil recovery performance. Hatiboglu and Babadagli (2008) performed static experiments (no flow in fracture) to simulate matrix-fracture interaction during miscible solvent injection in naturally fractured reservoirs. The authors showed that the displacement pattern, i.e., bulk diffusion and free convection, is highly affected by the interaction type (co- or counter-current), oil type, and displacement direction (horizontal and vertical). Kahrobaei et al. (2012) conducted static first contact miscible experiments using different oil-solvent pairs to take into account the effect of physical properties of solvent and oil on the recovery performance. Their results showed that at low oil viscosity and high solvent viscosity both diffusion and gravity forces are important and the recovery process is fast; however, at higher oil viscosity the recovery process is dominated by gravity.

Although there are a number of experimental and theoretical studies reported in the literature to examine the efficiency of miscible flooding in fractured reservoirs under static conditions, only a few of them have addressed the transfer mechanisms between matrix and fracture under dynamic (flow in fracture) conditions (Firoozabadi and Markeset 1994, Jamshidnezhad et al. 2004, Trivedi and Babadagli 2008, Verlaan and Boerrigter 2006). In addition, most of the dynamic experimental studies deal with the condition that the density and viscosity of the solvent is less than that of the oil in the matrix for practical purposes. Consequently, a detailed analysis of the transfer mechanisms via comparing the experimental data with transport models under dynamic conditions is missing in the literature. The main intent of this paper is to investigate the factors that affect the performance of miscible solvent flooding in fractured reservoirs at non-zero flow conditions for various oil/solvent viscosities and density ratios, and injection rates. To visualize and quantify the process, a CT-scan was used to investigate the transport phenomena in three-dimensional porous media (Farajzadeh et al. 2011, Simjoo et al. 2012, Simjoo et al. 2011).

This paper is aimed at (a) visualizing mass transfer process under flow conditions between matrix and fracture using a CT-scanner, (b) calculating the solvent and oil concentrations in the matrix using the 


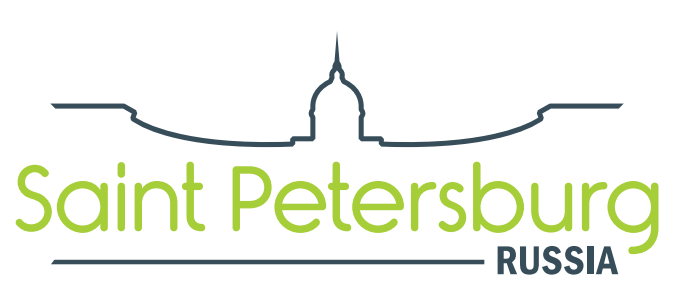

obtained CT data to calculate the oil recovery at different times, and (c) understanding the underlying oil recovery mechanisms in miscible solvent injection in fractured reservoirs using the experimentally obtained data and numerical simulations. The structure of the paper is as follows: the applied experimental method and the materials used in this study are explained in Section 2. Thereafter, the experimental results are presented and discussed in Section 3. The comparison between experimental data and simulation results is given in Section 4. Finally, the paper is ended with concluding remarks.

\section{Experimental work}

The experimental set-up consists of three major parts (1) an X-ray transparent core holder made from polyether ether ketone (PEEK) polymer; the core holder has an inner diameter of $4.52 \mathrm{~cm}$ and its length is $12.0 \mathrm{~cm}(2)$ an injection pump to inject the solvent at constant flow rate (3) a CT-scanner to take images at different times. A schematic diagram of the setup is shown in Figure 1. The empty space between the core and the wall of the core holder mimics a fracture. Two thin slits $(0.5 \mathrm{~mm})$ also exist between top and bottom side of the core and the core holder. This means all sides of the core are exposed to solvent while solvent is injected through the fracture. Experiments were conducted with seven different core plugs. The core plugs differed in size, viz. core plugs of length $11.90 \mathrm{~cm}$ with a diameter $d_{1}=4.00 \mathrm{~cm}$ and $d_{2}=4.44 \mathrm{~cm}$ respectively. This makes it possible to study the effect of solvent velocity in the fracture, while the injection rate is the same for the two different core diameters. The permeability of Bentheimer sandstone core (high permeable) was $1.44 \times 10^{-12} \mathrm{~m}^{2}(1.46$ D). The permeability of low permeability core was $2.46 \times 10^{-13} \mathrm{~m}^{2}(250 \mathrm{mD})$. The porosity of both sandstone cores were $0.21 \pm 0.01$. For each of the seven experiments a different core plug was used drilled from the high perm block and low perm block respectively. All experiments were conducted at room temperature $\left(20^{\circ} \mathrm{C}\right)$ and 2.0 bar backpressure. In each experiment different oil-solvent pairs were used. The properties of the model oils and solvents are listed in Table 1.

Table 1 Properties of the model fluids used in the experiments

\begin{tabular}{lllcc}
\hline Material & Chemical formula & Type & Density $\left[\mathrm{kg} / \mathrm{m}^{3}\right]$ & Viscosity [cp] \\
1-iodohexadecane & $\mathrm{C}_{16} \mathrm{H}_{33} \mathrm{I}$ & doping agent & 1120.7 & 8.430 \\
Hexane & $\mathrm{C}_{6} \mathrm{H}_{14}$ & oil/solvent & 654.8 & 0.294 \\
Pentane & $\mathrm{C}_{5} \mathrm{H}_{12}$ & solvent & 626.0 & 0.239 \\
Petroleum Ether & $\mathrm{CAS}$ No. 64742-49-0 & solvent & 651.1 & 0.292 \\
Hexadecane & $\mathrm{C}_{16} \mathrm{H}_{34}$ & oil & 773.38 & 3.447 \\
Squalane & $\mathrm{C}_{30} \mathrm{H}_{62}$ & oil/solvent & 810.1 & 36.00 \\
Hexadecane+doping & -------- & oil & 824.48 & 3.901 \\
Squalane+doping & ------- & oil & 857.5 & 25.75 \\
\hline
\end{tabular}

$\S$ densities and viscosities were calculated using the mixing rules given in Appendix.

For the acquisition of the CT images, the oil phase was doped with $20 \mathrm{wt} \%$ of 1 -Iodohexadecane. The addition of 1-Iodohexadecane to the oil phase improves the X-ray attenuation contrast between the oil and solvent phases, which leads to a clear contrast between the fluids in the CT scans. Note that addition of 1-Iodohexadecane changes, either decrease or increase, the density and viscosity of the model oil. At the beginning of each experiment the core was positioned vertically in the core holder. Then the core was vacuumed at least for 24 hours to remove the air from the porous medium. Thereafter, oil was injected from the bottom of the core holder to saturate the core fully with oil. A CT-scan was made from the $100 \%$ saturated core to obtain the CT-number of the oil. Subsequently, solvent was injected at constant flow rate and CT scans were taken frequently from the cross section of the core to record the changes with time. Assuming conservation of fluid volume, it is possible to show that the amount of produced oil from each pixel at each time step is equal to the saturation of the solvent in the same pixel in the core, which in turn can be related to the corresponding CT numbers. We obtain:

$$
\% \text { solvent inside a pixel }=\% \text { produced oil from the pixel }=\frac{C T_{\text {exp. }}-C T_{\text {oil }}}{C T_{\text {solvent }}-C T_{\text {oil }}},
$$




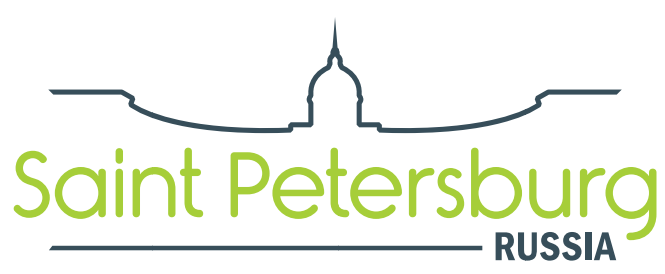

where $C T_{\text {oil }}$ is the CT-number of a pixel inside a fully saturated core with oil. $C T_{\text {solvent }}$ is the CTnumber of the same pixel in case of the fully saturated core with solvent. $C T_{\exp }$ is the CT-number inside the same pixel in the core, during the experiment. The accuracy of the above equation is within $\pm 2 \%$ (Farajzadeh et al. 2009). Addition of all pixel contributions and assuming radial symmetry of the concentration profile give the production of oil from the core. Table 2 summarizes the experiments conducted in this study.

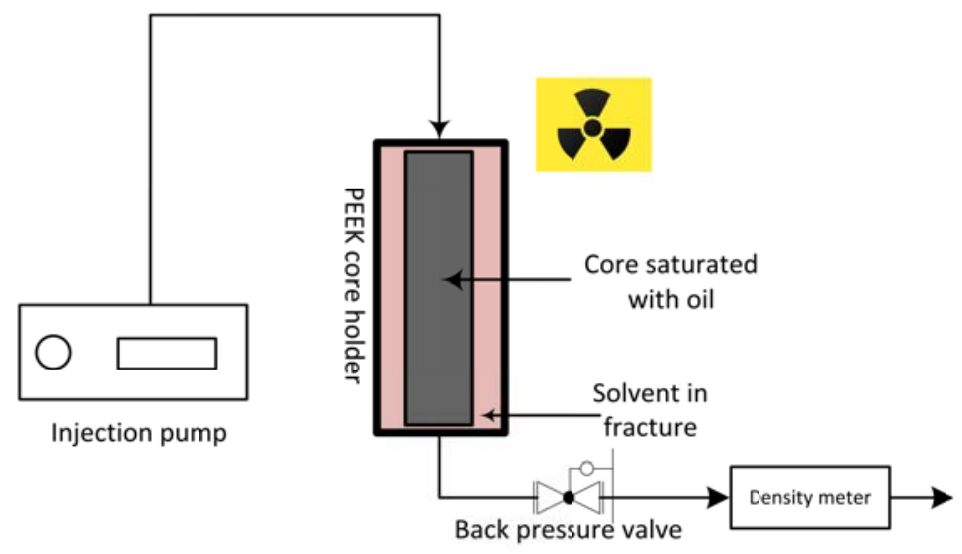

Figure 1 Schematic of the experimental setup. Not to scale.

Table 2 Detailed outline of experiments conducted

\begin{tabular}{ccccccc}
\hline $\begin{array}{c}\text { Exp. } \\
\#\end{array}$ & $\begin{array}{c}\text { Permeability } \\
{[\mathrm{D}]}\end{array}$ & $\begin{array}{c}\text { Pore volume } \\
{[\mathrm{ml}]}\end{array}$ & $\begin{array}{c}\text { Fracture aperture } \\
{[\mathrm{mm}]}\end{array}$ & Oil phase & Solvent & $\begin{array}{c}\text { Flow rate } \\
{[\mathrm{ml} / \mathrm{min}]}\end{array}$ \\
\hline 1 & 1.46 & 31.5 & 2.6 & hexadecane & hexane & 0.1 \\
2 & 1.46 & 31.5 & 2.6 & hexadecane & hexane & 0.5 \\
3 & 1.46 & 31.5 & 2.6 & hexadecane & hexane & 2.0 \\
4 & 1.46 & 40.5 & 0.4 & hexadecane & hexane & 0.1 \\
5 & 1.52 & 31.5 & 2.6 & squalane & pentane & 0.1 \\
6 & 1.48 & 31.5 & 2.6 & hexane & squalane & 0.5 \\
7 & 0.250 & 31.5 & 2.6 & squalane & Petroleum Ether & 0.1 \\
\hline
\end{tabular}

\section{Experimental Results}

In this section the experimental results are described. As mentioned before, the pressure gradient along the core is very small and negligible. Moreover, there is no capillary force since the solvent and the oil in the core are first contact miscible. Consequently, the displacement mechanism is either due to the penetration of solvent into the matrix or due to the gravity forces or possibly both.

\subsection{Effect of flow rate}

Experiments 1-3 were conducted using hexadecane as model oil and hexane as solvent. For such a system, the oil phase (doped with $20 \mathrm{wt} \%$ of 1-Iodohexadecane) is almost 13 times more viscous than the solvent. The density ratio, i.e., oil density/solvent density, is 1.26 , which means that the oil phase is denser than the solvent. For each test a new high permeable core sample with $d=4.00 \mathrm{~cm}$ was used as porous medium (Table 2). The maximum (analytical) drainage rate can be calculated using Eq. (2) (Verlaan and Boerrigter 2006), i.e.,

$$
q_{o}=A k_{z} \lambda_{o} \Delta \rho_{\text {oil-solvent }} g \text {, }
$$

where $A\left(\mathrm{~m}^{2}\right)$ is the cross-sectional area, $k_{z}$ the vertical permeability $\left(\mathrm{m}^{2}\right), \lambda_{o}$ the oil mobility, $\Delta \rho$ $\left(\mathrm{kg} / \mathrm{m}^{3}\right)$ the density difference between oil and solvent, and $g\left(\mathrm{~m} / \mathrm{s}^{2}\right)$ the gravity constant. For the rockfluid system considered in Experiments 1-3, the maximum oil drainage rate $q_{\mathrm{o}}$ is calculated as: 


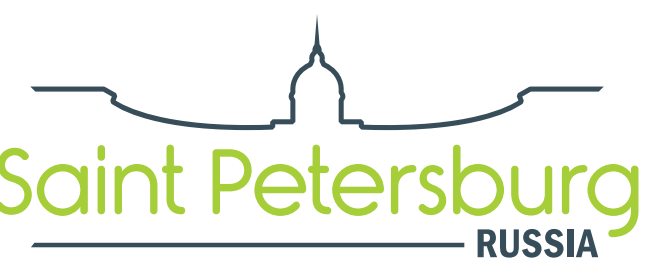

$$
q_{o}=\frac{\left(1.26 \times 10^{-3}\right)\left(1.46 \times 10^{-12}\right)(169.68)(9.8)}{3.901 \times 10^{-3}}=7.84 \times 10^{-10} \mathrm{~m}^{3} / \mathrm{s}=0.047 \mathrm{ml} / \mathrm{min},
$$

This means that all the injection rates used in Experiments 1-3 are higher than the analytically calculated drainage rate. The recovery profiles of hexadecane at three different injection rates, viz., $0.1,0.5$, and $2.0 \mathrm{ml} / \mathrm{min}$, are shown in Figure 2. A comparison between the recovery histories of Experiments 1-3, shows that the lowest injection rate (Experiment 1) has the lowest production rate. However, the difference in recovery efficiency of the other two cases, i.e., 0.5 and $2.0 \mathrm{ml} / \mathrm{min}$, is not significant. Note that the fractured system is initially filled with oil. Therefore, in case of low injection rate, it takes a long time to drain all the oil from the fracture and expose the whole length of the core to gravity drainage. It should be mentioned that the recovery factor was calculated based on the volume of oil drained from the matrix. The velocity $V$, (residence time of solvent in the fracture $L / V$ ), can be calculated using

$$
V=\frac{q_{i n j}}{A}=\frac{q_{i n j}}{\pi \times\left(r_{\text {coreholder }}^{2}-r_{\text {core }}^{2}\right)},
$$

where $A$ is the area open for flow in the fracture and the porosity in the fracture is one. According to Eq. (4), the residence time of solvent in fracture for the low injection rate case (Experiment 1) is about 417 minutes. For the increased solvent velocity in the fracture (Experiments 2 and 3), the residence time in fracture decreases. The residence time of solvent in the fracture for Experiment $2(0.5 \mathrm{ml} / \mathrm{min})$ and Experiment $3(2 \mathrm{ml} / \mathrm{min})$ are 83.5 and 20.87 minutes, respectively (see equation 4). For Experiment 1, the experimental production rate is smaller than the analytical drainage rate while the injection rate is higher than the analytical drainage rate. This is due to the fact that the oil-solvent interface in the fracture system moves downward very slowly and production from the matrix can keep up with the solvent/oil interface in the fracture. Consequently only a small portion of the core length is exposed to gravity drainage forces (Figure 3a). In case of experiments 2 and 3, the production rates are almost the same, but now gravity drainage is more important. Indeed, the rates are the same within experimental accuracy. The small difference can be correlated to the uncertainty in image analysis and fluid saturation calculations. This clearly implies that for the condition that the oil-solvent interface moves down faster in the fracture, the proportion of the core that will experience gravity drainage increases and therefore the drainage rate increases. In the low injection rate experiment, i.e., Experiment 1, the production rate is limited by the injection rate, and by increasing the injection rate in Experiments 2 and 3, this restriction is removed. Thus, it can be concluded that the solvent injection rate is the rate-determining process for the oil production. It should be mentioned that due to the high volume of solvent needed to produce oil from the matrix, Experiment 3 was stopped after 150 minutes.

A major concern for miscible fluid injection in fractured oil reservoirs is the early breakthrough and production of large quantities of the injected fluid. Higher injection rates through the fracture system leads to earlier breakthrough and therefore solvent has less time to exchange mass with the oil that resides in the matrix. When the injection rate is reduced, the breakthrough time of the solvent from the fracture is postponed and consequently the solvent has more time to extract oil from the matrix. However, as shown in Figure 2, a lower injection rate corresponds to less solvent per unit time per unit volume of oil in the matrix. From an economical point of view, one may prefer to use less solvent per unit volume of oil produced. According to Figure 2, lowering the flow rate makes it possible to produce all the oil in place using less solvent. However, the lower flow rate in the fracture leads also to a lower production rate. In summary, the efficiency of a miscible solvent process can be assessed with respect to either the production rate or the volume of oil produced per unit volume of solvent injected. Figure 4 shows the recovery of hexadecane from Experiments 1-3 versus the amount of solvent injected (PV). It can be concluded from Figure 4 that for faster recovery, i.e., higher drainage rates, the process should be performed at higher injection rates. However, this costs more solvent. On the other hand, if the amount of solvent injected is the critical parameter, the process should be performed at lower flow rates. Therefore, it is of practical importance to define a critical velocity 


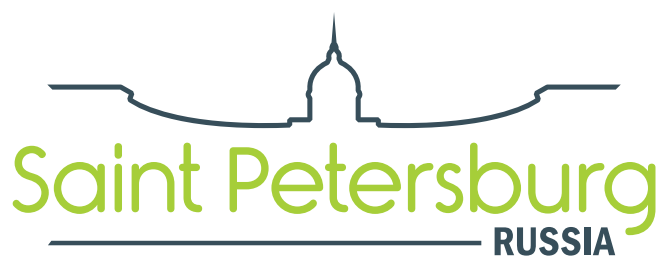

which can be correlated to the matrix, fracture and fluid properties. This makes it possible to evaluate and optimize the economy and efficiency of the process regarding the operating conditions.

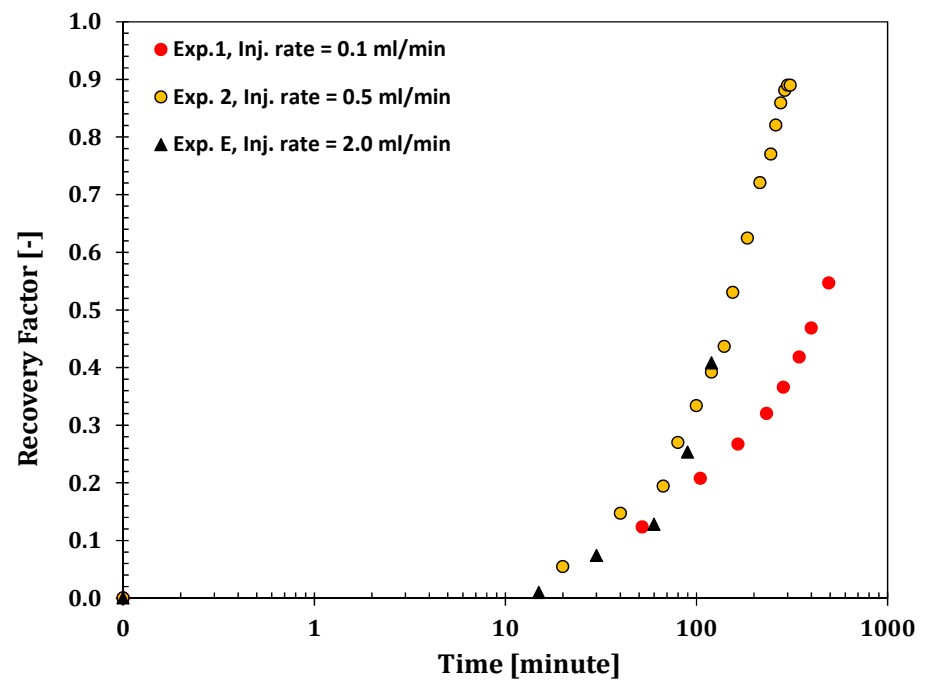

Figure 2 Recovery from matrix vs. time at different flow rates viz., 0.5, 0.1, and $2.0 \mathrm{ml} / \mathrm{min}$.

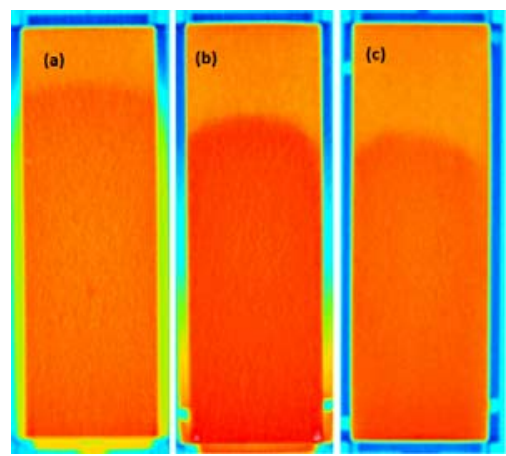

Figure 3 Position of oil-solvent interface in the fracture at different injection flow rates and after 100 minutes injection (a) $0.1 \mathrm{ml} / \mathrm{min}$, (b) $0.5 \mathrm{ml} / \mathrm{min}$, and (c) $2.0 \mathrm{ml} / \mathrm{min}$. For higher flow rates, the interface in the fracture moves downward faster. Consequently a larger part of the core is exposed to gravity forces for higher flow rates.

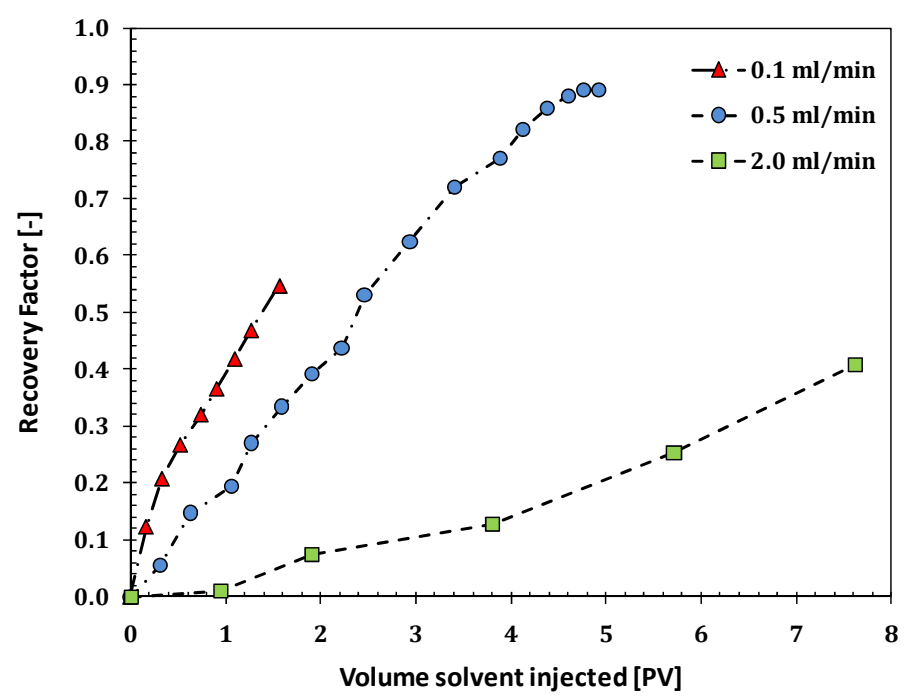

Figure 4 Recovery from the matrix vs. pore volume solvent injected at different flow rates viz., 0.5, 0.1 , and $2.0 \mathrm{ml} / \mathrm{min}$. For higher flow rates more solvent should be used to recover oil from the matrix. 


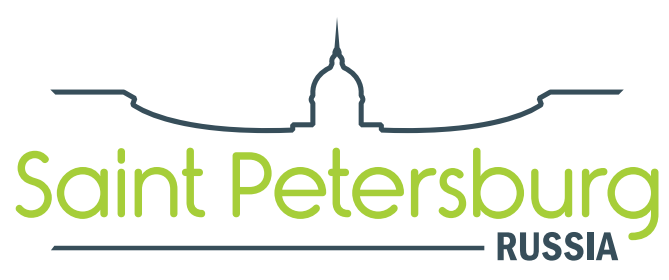

Figure 5 shows the CT images of Experiment 1 at different time steps. Note that the oil is shown in red and the solvent is in blue. Figure 5 shows that the solvent invades the matrix mainly from the top and the oil is produced through the fracture. In this case, a sharp stable front moves downward. Based on the results, a lower injection rate enhances the stability of the front. This is due the fact that the production in the matrix, which determines the position of the solvent-oil interface in the matrix, is restrained by the low injection rate and thus by the solvent/oil interface in the fracture.

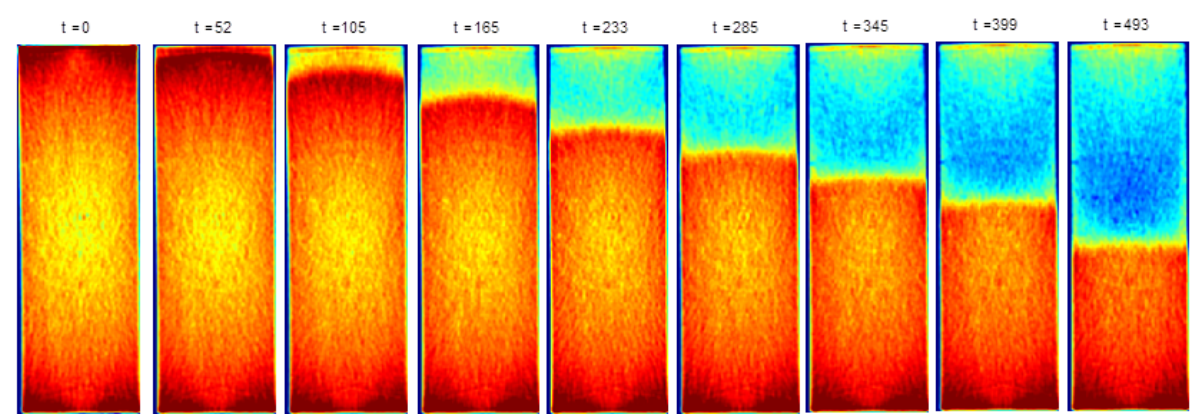

Figure 5 Process visualization of Experiment 1 (hexadecane-hexane system at $0.1 \mathrm{ml} / \mathrm{min}$ injection rate). Time above CT image is in minutes.

Figure 6 shows the CT images of Experiment 2 at different time steps. The oil phase is shown in red and the solvent is in blue. From Figure 6, the solvent starts to penetrate into the core from the top. However, the invasion is not uniform and leads to a skewed shape profile. This can be attributed to the heterogeneity of the core. Moreover, CT images were taken only from the central slice of the core. In other words, it is assumed that the system is symmetric in the radial direction. However, the production profile might be different in each slice of the core due to the gradient of solvent concentration in radial direction. Consequently, the displacement may not be accurately described using a 2-D image and high resolution full 3D images would be needed to capture these instabilities. This is part of our ongoing research. The curved shape of the moving front is attributed to the fact that in a gravity drainage situation, where mixing at the solvent/oil interface takes place (yellow in CT images), the oil viscosity and density are both reduced. In the mixing zone between solvent and oil, the drainage rates are higher than in the oil zone (red) and the excess is flowing sideways to the fracture. The viscosity reduction effect on the drainage rate is therefore larger than the density reduction effect (Verlaan and Boerrigter 2006).
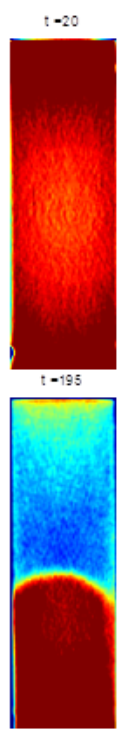
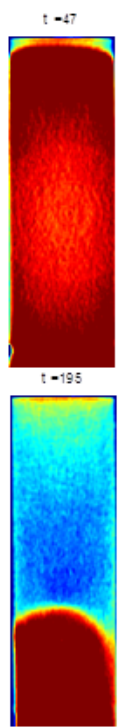
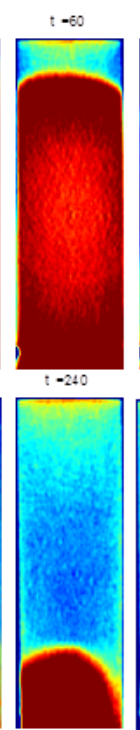
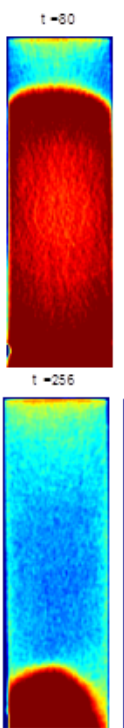
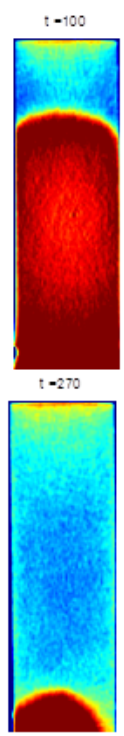

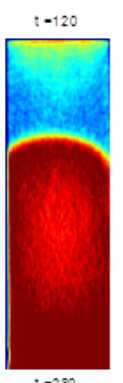

$t=200$

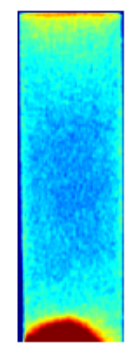

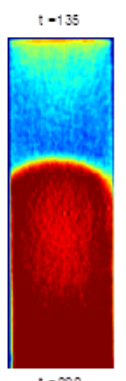
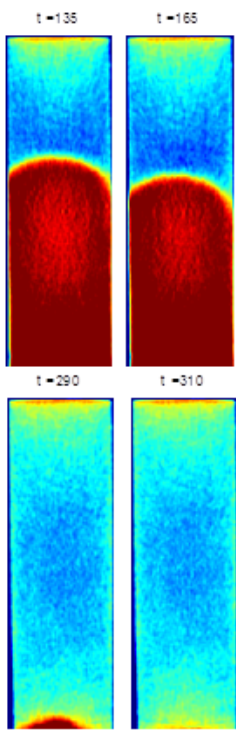

Figure 6 Process visualization of Experiment 2 (hexadecane-hexane system at $0.5 \mathrm{ml} / \mathrm{min}$ injection rate) using CT-scan. Time above CT image is in minutes. 


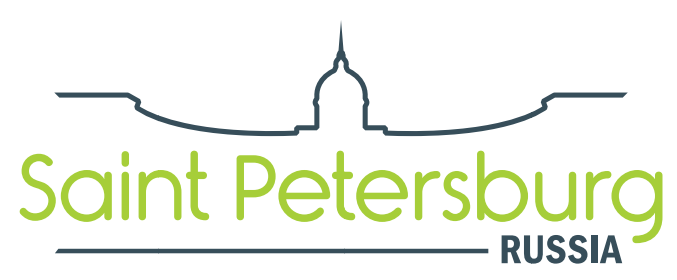

Figure 7 shows the CT images of Experiment 3 at different times. It can be seen from Figure 5 that a relatively stable front moves downward similar to the case with the lower flow rate, i.e., Figure 6. For early times, the solvent penetrates into the core mainly from the top. Again, the skewed shape of the moving interface is attributed to the fact that the drainage rate at the interface (yellow) is higher than the analytical drainage rate due to the diffusion and dispersive flow inside the matrix. Therefore, the excess production flows to the fracture and leads to a curved shape profile. Comparing between CTimages of Experiment $2(0.5 \mathrm{ml} / \mathrm{min})$ and Experiment $3(2.0 \mathrm{ml} / \mathrm{min})$ at almost the same time (i.e., after 100 minutes of solvent injection) reveals that an increasing flow rate has no significant effect on the production profile as the injection rate is above the drainage rate at the mixing zone and the whole core is already exposed to the gravity forces. In other words, the fracture system is kept fresh with the solvent and therefore the excess produced oil is drained through the fracture system.
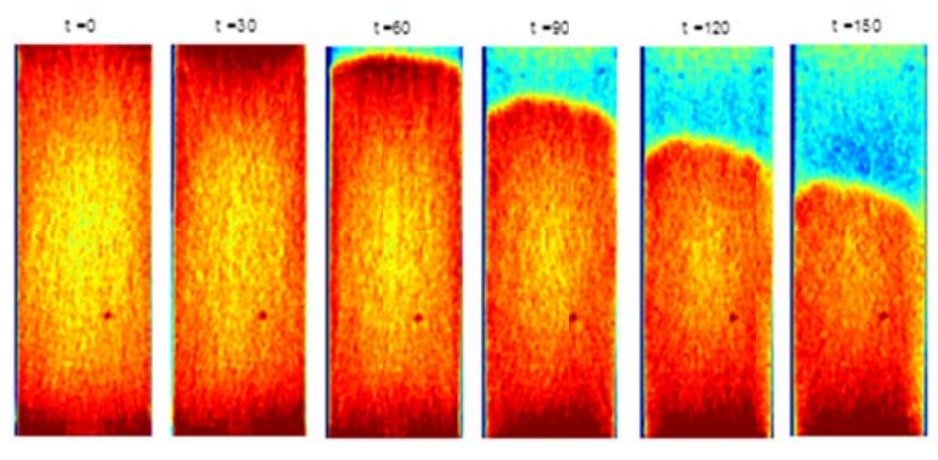

Figure 7 Process visualization of Experiment 2 (hexadecane-hexane system) with CT-scan. Injection rate $2.0 \mathrm{ml} / \mathrm{min}$, cf. Figure 2. Time above CT image is in minutes.

\subsection{Effect of fracture aperture}

Based on the results of Experiments 1-3, it can be concluded that the production profile vs. time is influenced by the injection rate of the solvent. For a given fracture aperture, different injection rates lead to different solvent velocities, i.e., different fluxes of solvent flowing through the fracture per unit time. Changing the fracture aperture, while keeping the injection rate constant, makes it possible to change the solvent velocity through the fracture at a constant volumetric injection rate. Experiment 4 was conducted to examine the effect of fracture aperture on the production profile of hexadecane from the matrix using hexane as solvent. The injection rate was set at $0.1 \mathrm{ml} / \mathrm{min}$, the same as in Experiment 1. A high permeability core of $4.44 \mathrm{~cm}$ diameter was used as porous medium. In this case the fracture aperture is $0.4 \mathrm{~mm}$ (400 micrometer), which leads to a solvent velocity of $0.177 \mathrm{~cm} / \mathrm{min}$ through the fracture. This velocity corresponds to a residence time of 67 minutes in the fracture system. Figure 8 shows the recovery history of hexadecane obtained from Experiments 1 and 4 . According to Figure 8, after 100 minutes the recovery curve of Experiment 4 overtakes the recovery curve of Experiment 1 and leads to higher production rates compared to experiment 1 . The difference between production histories can be attributed to the fact that in case of lower velocity in the fracture, i.e., Experiment 1, it takes a longer time to displace the oil from the fracture, hence exposing the full core to the gravity drainage gradient. While, in the case of higher velocity, i.e., Experiment 4, the whole core is exposed to gravity drainage in a shorter time. Consequently, the production rate increases with time. Note that the amount of solvent that is injected through the fracture is the same for Experiment 1 and Experiment 4.

Figure 9 illustrates the CT images of Experiment 4 at different times. The solvent invades the core from the top and a stable front moves downward until all the oil is produced. Comparing Figures 5 and 9 reveal that a low injection flow rate process leads to developing a stable front in the matrix, which moves downward until all the oil is produced. This is attributed to the fact that for low injection rate process, the injection rate is the controlling mechanism and the solvent in the fracture system is mixed with the oil from the matrix. A higher production from the matrix can preserve the solvent-oil interface in the fracture system and thus reduce the gravity drainage potential. The effect of fracture 


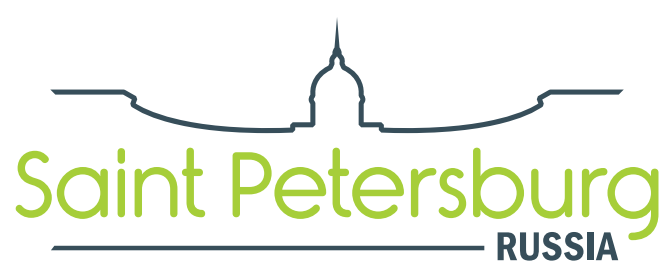

aperture needs to be addressed in more detail for a case that the injection rate is not the controlling mechanism of the process and this is part of our on-going research.

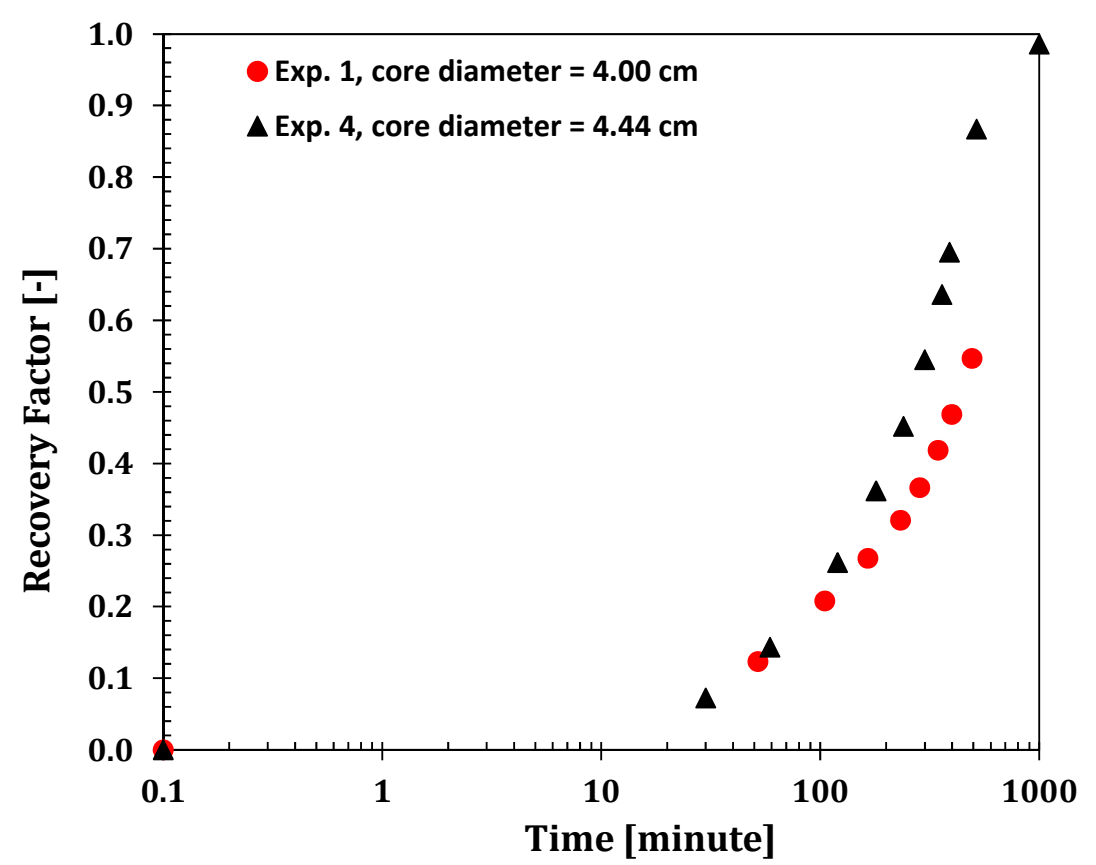

Figure 8 Recovery history of two core samples with different diameters. viz., 4.00 and $4.44 \mathrm{~cm}$, i.e., different fracture apertures $(2.6 \mathrm{~mm}$ and $0.4 \mathrm{~mm})$. The injection rate is constant at $0.1 \mathrm{ml} / \mathrm{min}$ for both cases.
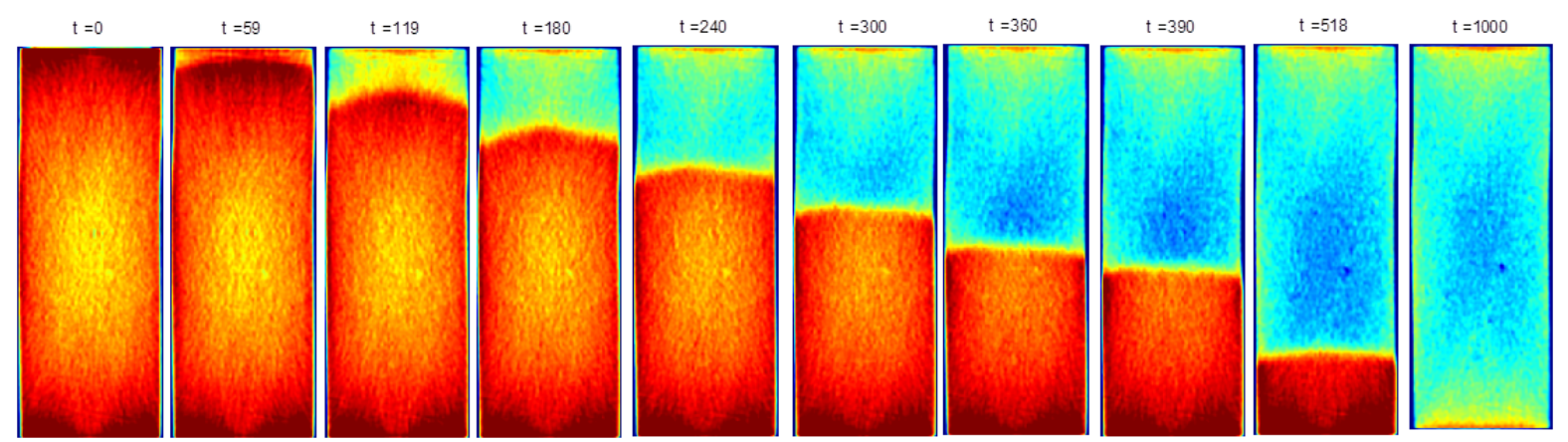

Figure 9 Process visualization of Experiment 4 (hexadecane-hexane system). Core diameter 44.4 $\mathrm{mm}$. Injection rate $0.1 \mathrm{ml} / \mathrm{min}$. Time above CT image is in minutes.

\subsection{Effect of oil-viscosity}

Miscible solvent injection is one of the efficient methods for heavy oil recovery. Therefore, it is of practical importance to analyze the effect of oil viscosity on the performance of miscible solvent injection in NFRs. Experiment 5 was performed using Squalane as model oil and n-pentane as solvent. In this case and for the conditions considered, the model oil (doped with $20 \mathrm{wt} \% 1$ iodohexadecane) is about 107 times more viscous than the solvent (see Table 1). The density ratio, i.e., oil density/solvent density, is 1.36. In this experiment a core with permeability of $1.52 \mathrm{D}$ and diameter of $4.00 \mathrm{~cm}$ was used as porous medium. The injection rate was set at $0.1 \mathrm{ml} / \mathrm{min}$. The recovery history of the experiment is shown in Figure 10. For the sake of comparison, the recovery profile of hexadecane with the same solvent injection rate (Experiment 1) is also shown in Figure 10. Note that the solvents used for these two experiments are different, namely n-hexane for Experiment 1 and n-pentane for Experiment 5. However, the viscosities and densities of these two solvents are 


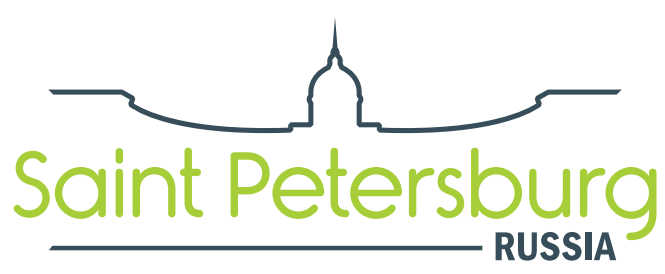

similar to each other. Hence, we do not expect an effect of the type of the solvent. We do expect an effect of the oil, because of its large difference in viscosity. According to Figure 10, however, the recovery history is more or less the same in spite of the fact that the viscosity of Squalane is nearly 6.6 times higher than for hexadecane. This indicates that miscible solvent injection is an efficient method for viscous oils if the injection rate is not limiting. However, more experiments with highly viscous oils are needed to support this idea. It is expected that for very highly viscous oils, diffusion is the controlling mechanism.

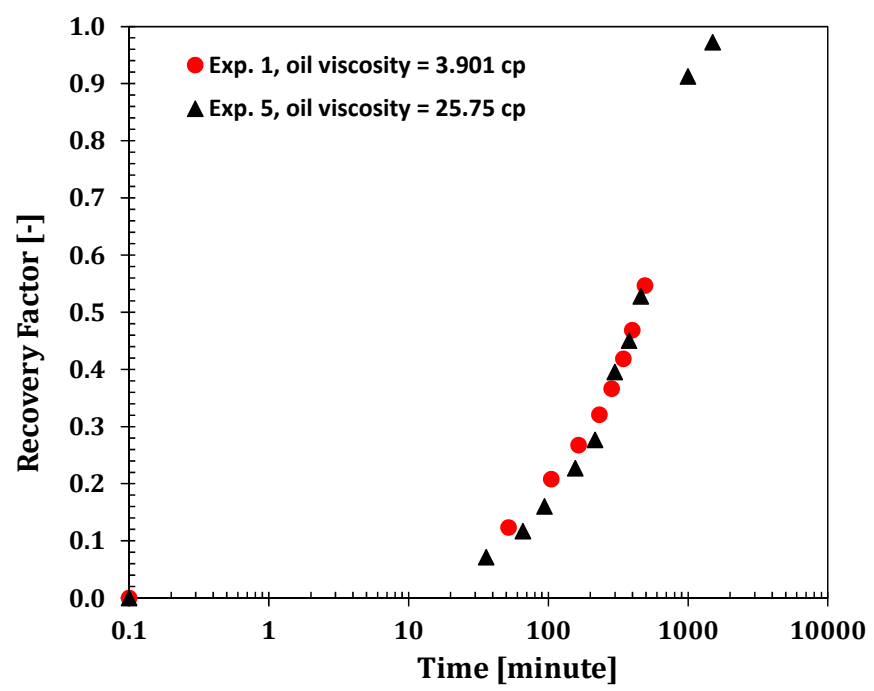

Figure 10 Recovery histories of Experiments 1 and 5. In this study the effect of oil viscosity is small.

Figure 11 shows the CT scans of Experiment 5 at different times. It is observed that the solvent invades the matrix mainly from the top. However, as injection continues, the shape of the downward moving front becomes increasingly concave. This is again an indication that the drainage rate in the mixing zone (yellow) is higher than in the oil zone (red) and consequently also higher than the amount of oil that spills at the interface from the matrix flux into the fracture system. In the other words, the viscosity reduction effect is larger than the density reduction effect. A comparison between Figures 5 and 10 shows that the viscosity reduction effect is more important in case of a more viscous oil as in Figure 11 the downward moving front becomes increasingly concave while in Figure 5 the front is almost flat.

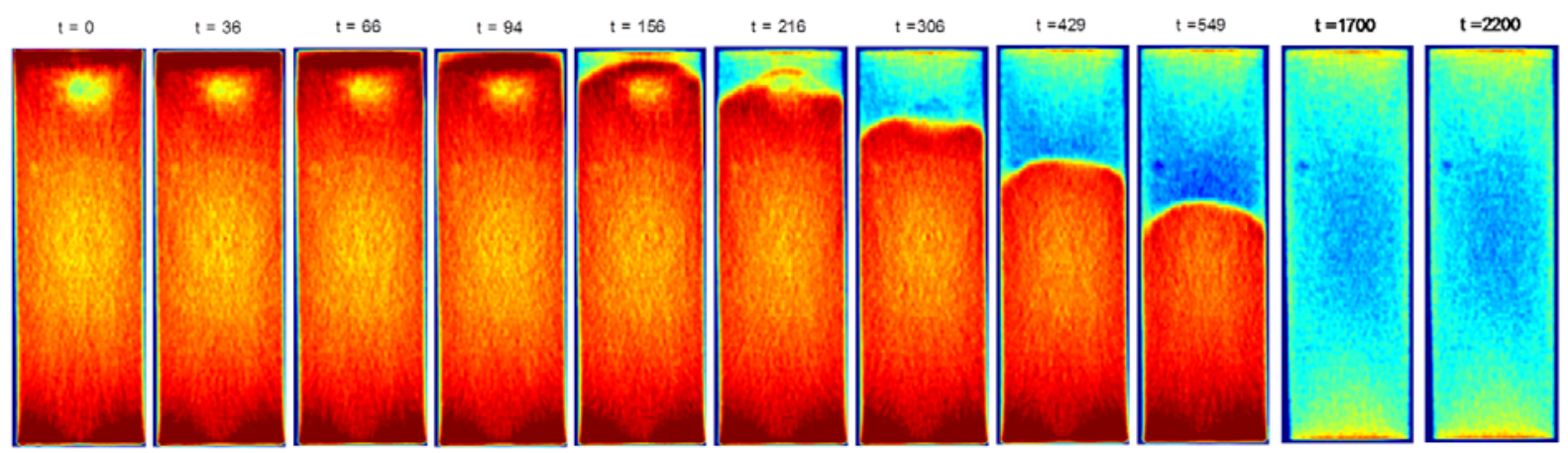

Figure 11 Process visualization of Experiment 5 (Squalane-Pentane system). Injection rate $0.1 \mathrm{ml} / \mathrm{min}$. Time above CT image is in minutes.

\subsection{Effect of oil/solvent density ratio}

Experiment 6 was performed using Squalane as solvent and n-hexane as the model oil. In this case, the solvent is heavier than the model oil. Moreover, the solvent is more viscous than the oil that resides in the matrix. This experiment is performed to understand the physics of the processes 


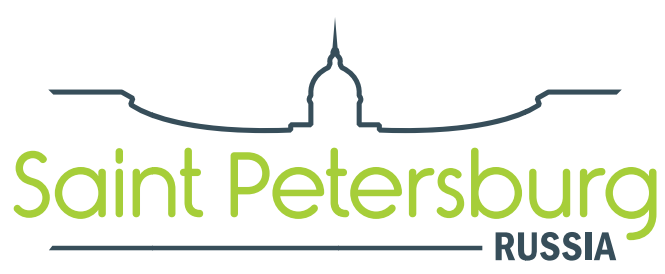

involved in miscible injection, as in practice solvents are typically less viscous than the oil. A high permeability core was used as porous medium. The injection rate from the top was set at $0.5 \mathrm{ml} / \mathrm{min}$. The recovery history of $n$-hexane is shown in Figure 12. This experiment is comparable to Experiment 5 except that Squalene is now used as solvent and hexane as the model oil. To make the comparison easier, the recovery histories of Experiments 5 and 6 are also plotted in Figure 12. According to Figure 12, it may be concluded that initially both cases behave the same, even though the arrangement of oil and solvent is different in these two cases. At later times, however, oil is produced faster in Experiment 6.

The CT images from Experiment 6 are shown in Figure 13. It can be observed that for the early time of solvent injection, the solvent penetrates the core from the top. However, after 132 minutes the solvent starts to invade the core also from the sides near the lower part of the core. Thereafter, the solvent penetrates into the core mainly from the sides and pushes oil out of the matrix. Therefore, at later stages, the production pattern in Experiment 6 is completely different from that of Experiment 5, i.e., the model oil (hexane in Experiment 6) is produced faster than the Squalane in Experiment 5. This can be attributed to the fact that, in Experiment 6, the solvent (Squalane) is heavier than the oil (hexane). When solvent is injected into the fracture, it flows rapidly downward due to gravity forces. Then, the solvent starts to exchange mass with the oil in the core, which is produced. From the CTscan data (Figures 11 and 13) it is observed that Experiment 5 shows stable behavior, but that Experiment 6 concerns an unstable displacement process.

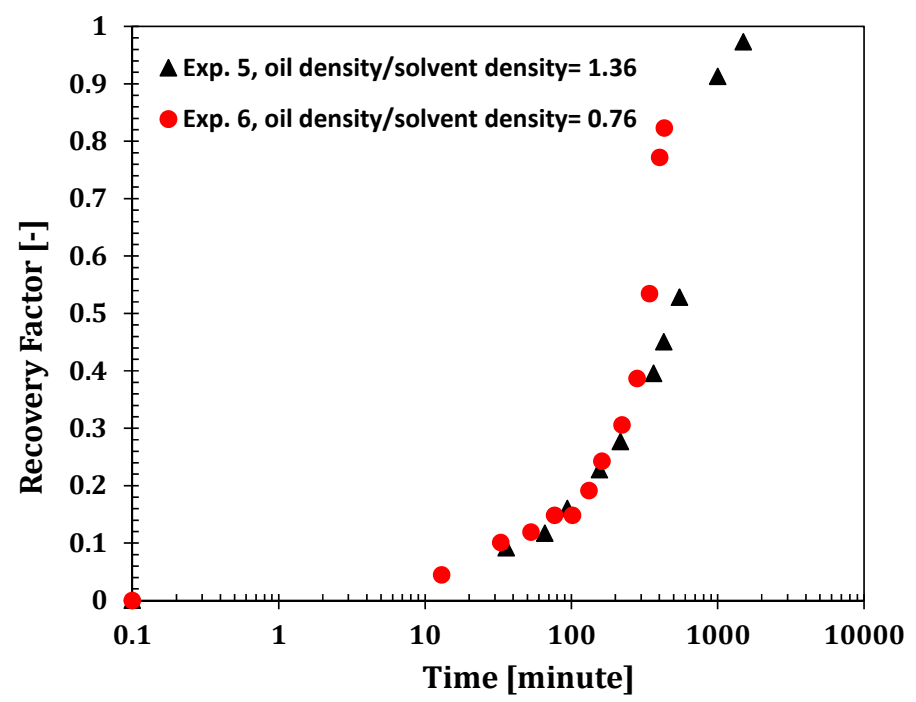

Figure 12 Recovery from the matrix in the hexane-Squalane system (Experiment 6). In the early stage the production rates behave similarly. However at later stages unstable penetration of Squalane occurs in Experiment 6 and enhanced transfer occurs.

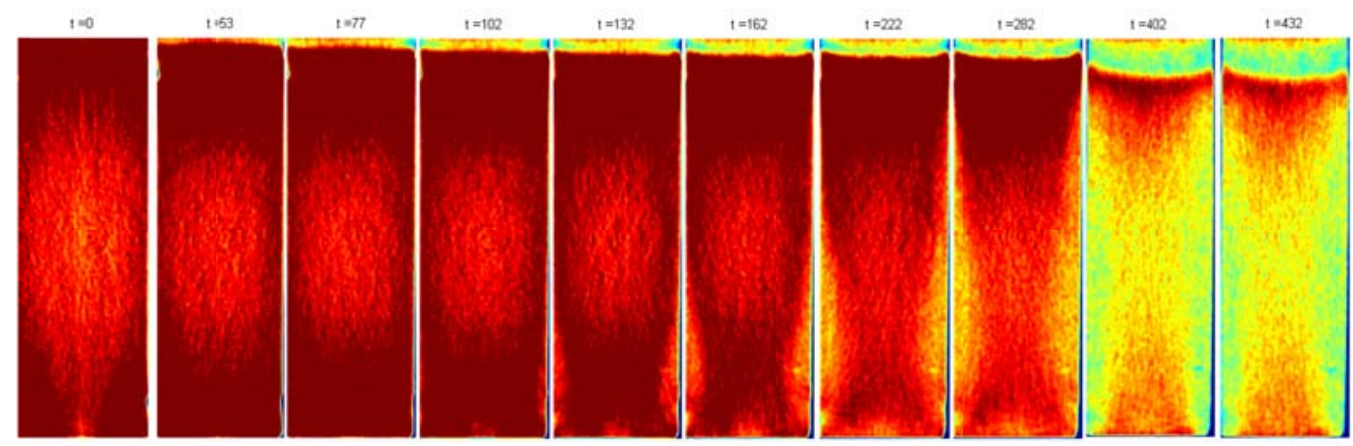

Figure 13 Process visualization of Experiment 6 (Hexane-Squalane system). Injection rate $0.5 \mathrm{ml} / \mathrm{min}$. Initially the process is very stable and there is little difference between experiments 5 and 6 . However at later stage enhanced (unstable) displacement of hexane by squalane occurs. 


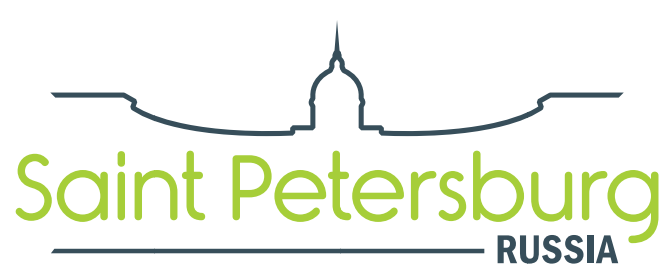

\subsection{Effect of matrix permeability}

Experiment 7 was conducted using a low permeable core as porous medium to examine the effect of matrix permeability on the production behavior of miscible solvent injection. In this experiment Squalene was employed as the model oil and petroleum ether as the solvent. Petroleum ether is composed of various volatile liquid hydrocarbons and is mainly used as a nonpolar solvent. The solvent was injected at a constant flow rate of $0.1 \mathrm{ml} / \mathrm{min}$. In this experiment the model oil is much more viscous than the solvent. This experiment is comparable with Experiment 5 except that now the matrix has a low permeability. Moreover, different solvents, yet with very similar properties, were used in these experiments. The recovery history of Squalane from Experiments 5 and 7 is shown in Figure 14. It is observed that for the low permeability rock, i.e., Experiment 7, diffusion is more important at the early times of solvent injection. However, after 400 minutes both cases show a similar production trend. The slow production rate during the early stages of Experiment 7 is attributed to the low permeability of the core sample. The CT scans of Experiment 7 at different times are shown in Figure 15. It can be observed that the downward moving front is not stable and some irregularities develop. Comparison between Figures 14 and 11 reveal that the instabilities of the moving front are more conspicuous in the case of a low permeability sample.

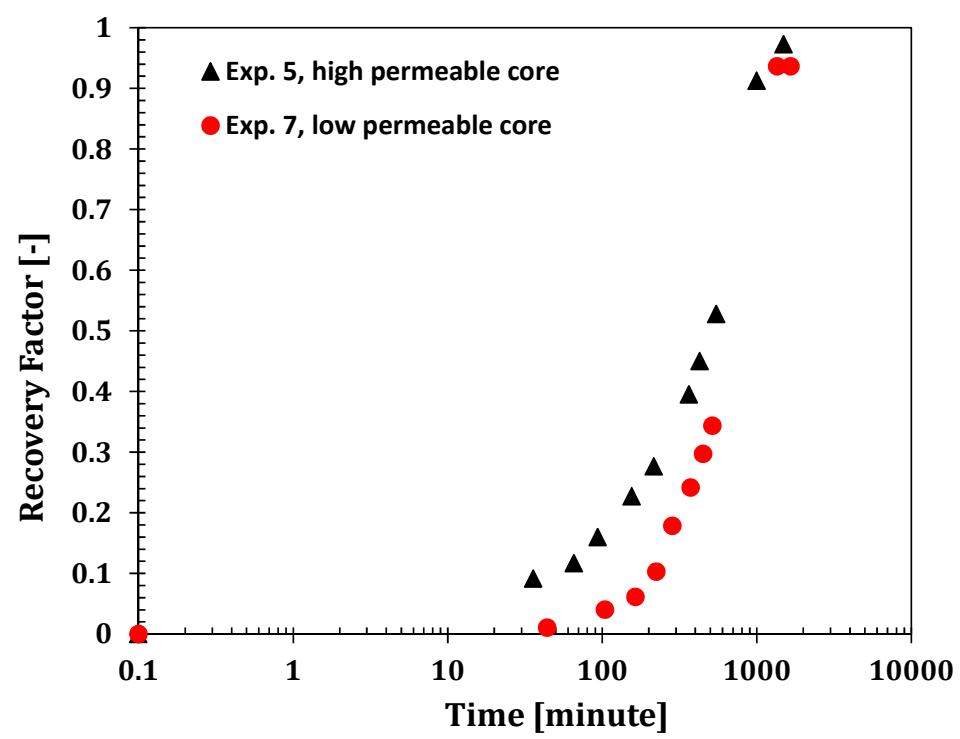

Figure 14 Recovery from the matrix in the Squalane-Petroleum ether system (experiment 7, low permeable core). Initially recovery is slower than in experiment 5; however after 600 minutes the recovery factor of experiment 7 becomes larger than of experiment 5 .
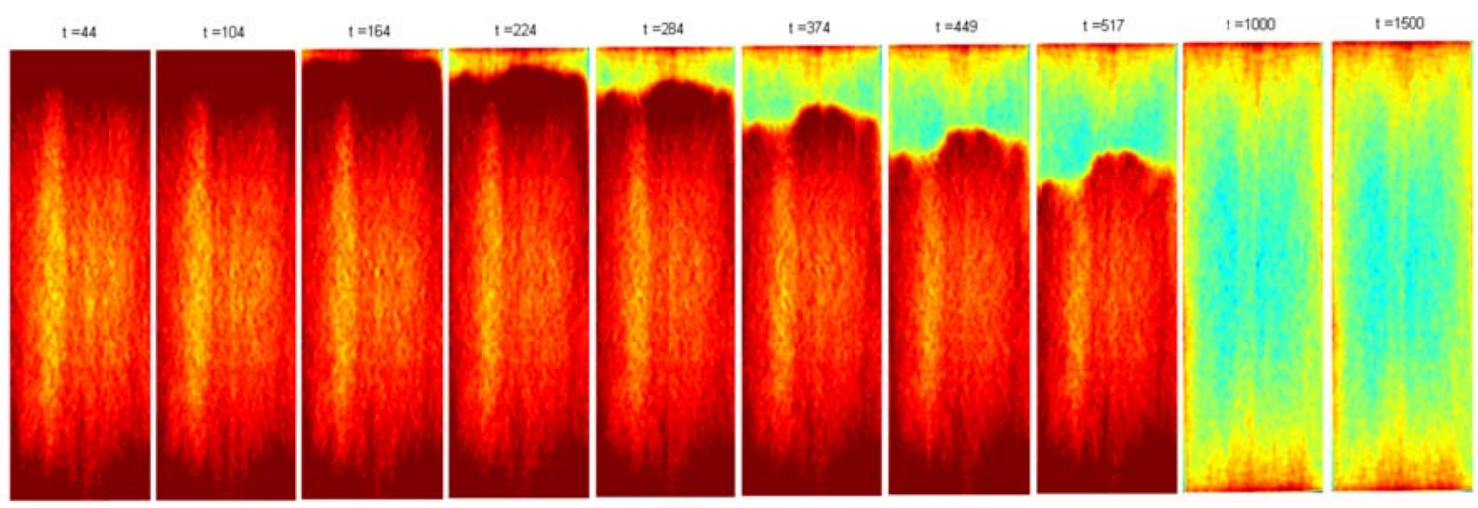

Figure 15 Process visualization of Experiment 7 (Squalane-Petroleum ether system). A low permeability core was used as porous medium. Injection rate $0.1 \mathrm{ml} / \mathrm{min}$. The interface shows irregular behavior; the irregularities increase with time. The times above the pictures are in minutes. 


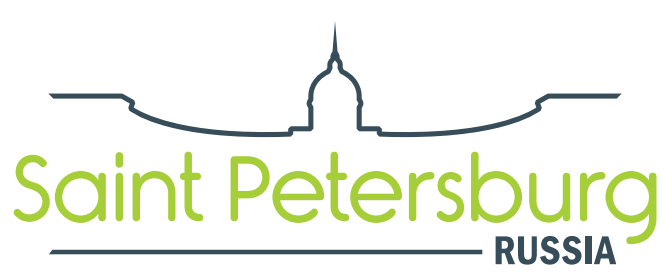

It can be inferred from Experiments 1-7, that the recovery performance and the production history of a miscible injection process depends on the injection rate and the volume of the solvent injected into the fracture amongst other parameters. Figure 16 illustrates the recovery histories of Experiments 1-4. From Figure 16, it seems that there is a potential production rate, above which the recovery history is no longer influenced by the injection rate.

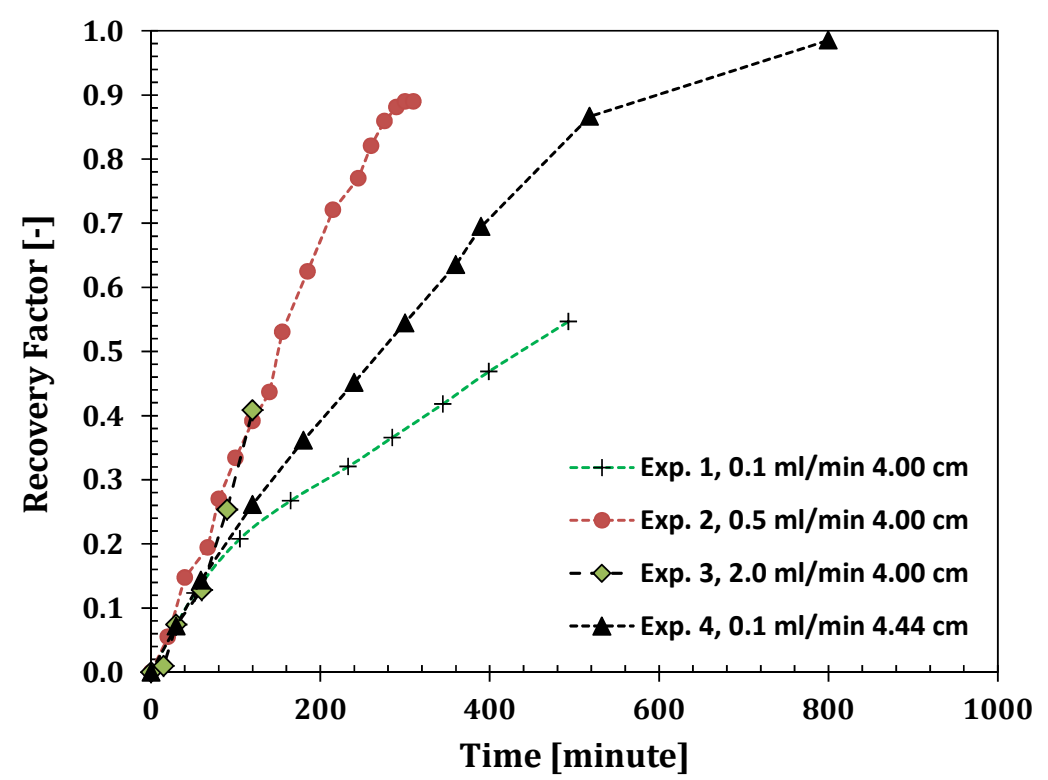

Figure 16 Recovery history of Experiments 1-4. The recovery history is not influenced by the injection rate as long as the injection rate is higher than the potential production rate.

\section{Model Equations}

The miscible injection process in a fractured porous medium is governed by the convection-diffusion equation (Berg et al. 2010, Tan and Firoozabadi 1995):

$$
\begin{gathered}
\nabla \cdot \mathbf{u}=0, \\
u=-\frac{k}{\mu}(\nabla p-\rho g), \\
\varphi \frac{\partial c}{\partial t}+\mathbf{u} . \nabla c=\nabla .(\varphi D \nabla c),
\end{gathered}
$$

where $\varphi$ is matrix porosity, $\mathbf{u}$ is Darcy velocity $(\mathrm{m} / \mathrm{s}), p$ is the pressure $(\mathrm{Pa}), k$ is the permeability $\left(\mathrm{m}^{2}\right)$, $\mu$ is the viscosity (Pa.s), and $\rho\left(\mathrm{kg} / \mathrm{m}^{3}\right)$ is the density. The density $\rho$ is assumed to depend linearly on the concentration (no volume contraction)

$$
\rho=\rho_{\text {sol. }} c+\rho_{\text {oil }}(1-c)=\rho_{\text {oil }}-\left(\rho_{\text {oil }}-\rho_{\text {sol. }}\right) c,
$$

The viscosity of the mixture is calculated from a log-linear viscosity mixing rule.

$$
\mu=\mu_{\text {oil }}^{x_{\text {oil }}} \cdot \mu_{\text {sol. }}^{x_{\text {sol. }}} \text {, }
$$

where $\mu_{o}$ is the viscosity of pure oil and $\mu_{\text {sol. }}$ is the viscosity of the solvent. Here, $x_{\text {oil }}$ and $x_{\text {sol }}$ are the mole fractions of oil and solvent, respectively. The relationship has been confirmed experimentally by Kahrobaei et al. (2012). Equations (5)-(9) were solved numerically using the reservoir simulation software packages MoReS (Shell in-house simulator) to numerically reproduce the experimental data. The numerical model represents an oil saturated porous medium surrounded by a small slit, which simulates the fracture. For the fracture, the local porosity was assumed to be $\varphi_{f}^{*}=1$ and a very high local permeability $\left(k_{f}^{*}=1000\right.$ Darcy) assigned to the fracture grid blocks. The dimensions in the 


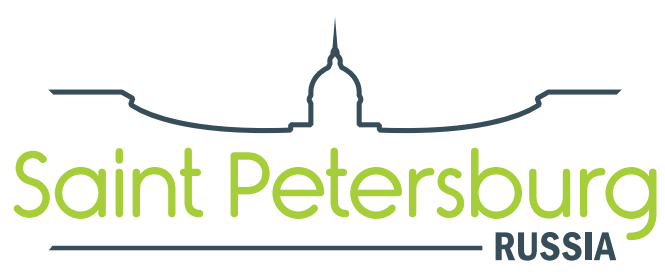

physical model are the same as those in the experiments. A radial symmetric grid was applied to solve the equation numerically using the finite difference method. Figure 17 shows the $R$ - $Z$ cross section of the simulation model. The injection and production wells were positioned in the first and last grid cells in $Z$ direction, respectively (see Figure 17). The solvent is injected at constant flow rate from the top and oil is produced at the same rate from the bottom. The area shown in green represents the solvent in the fracture and red area represents the core plug. In the model, both the core and fracture are initially saturated with the model oil. Thereafter, solvent is injected through the fracture at constant flow rate and recovery is calculated based on the volume of oil produced from the matrix alone.

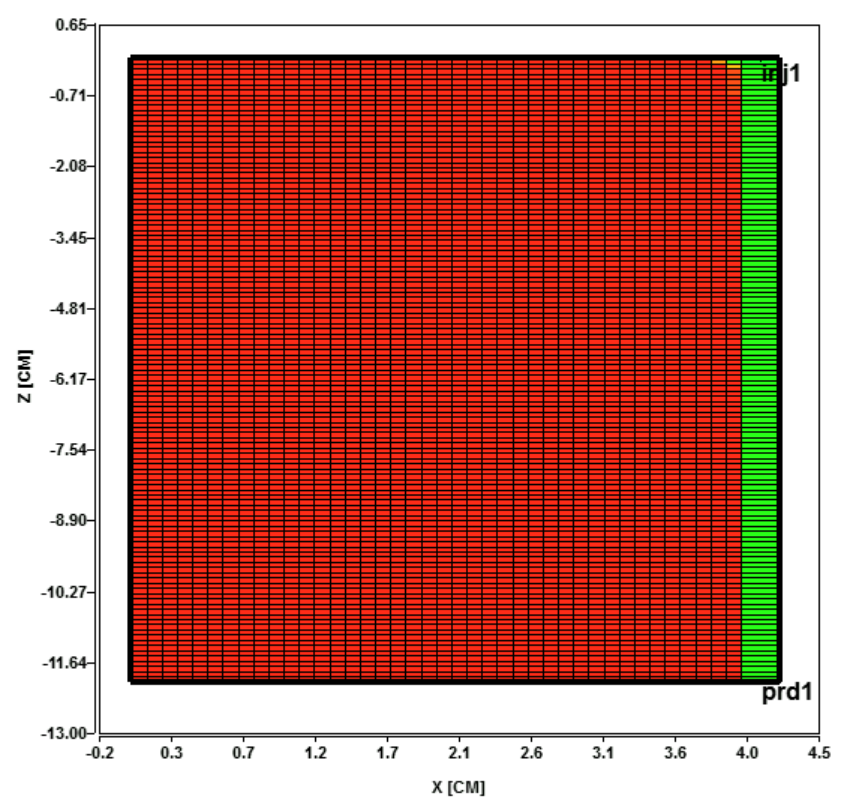

Figure $17 R-Z$ cross-sectional view of MoReS simulation model

4.1. Simulation of Experiment 1. The comparison between experimental and simulation results of Experiment 1 is shown in Figure 18. The simulation results are in good agreement with the experimental data. The mixing in the simulation is due to numerical dispersion only. Grid refinement sensitivity analysis revealed that a grid size of $1 \mathrm{~mm}$ should be considered to reduce the numerical dispersion and thus to reduce spurious production.

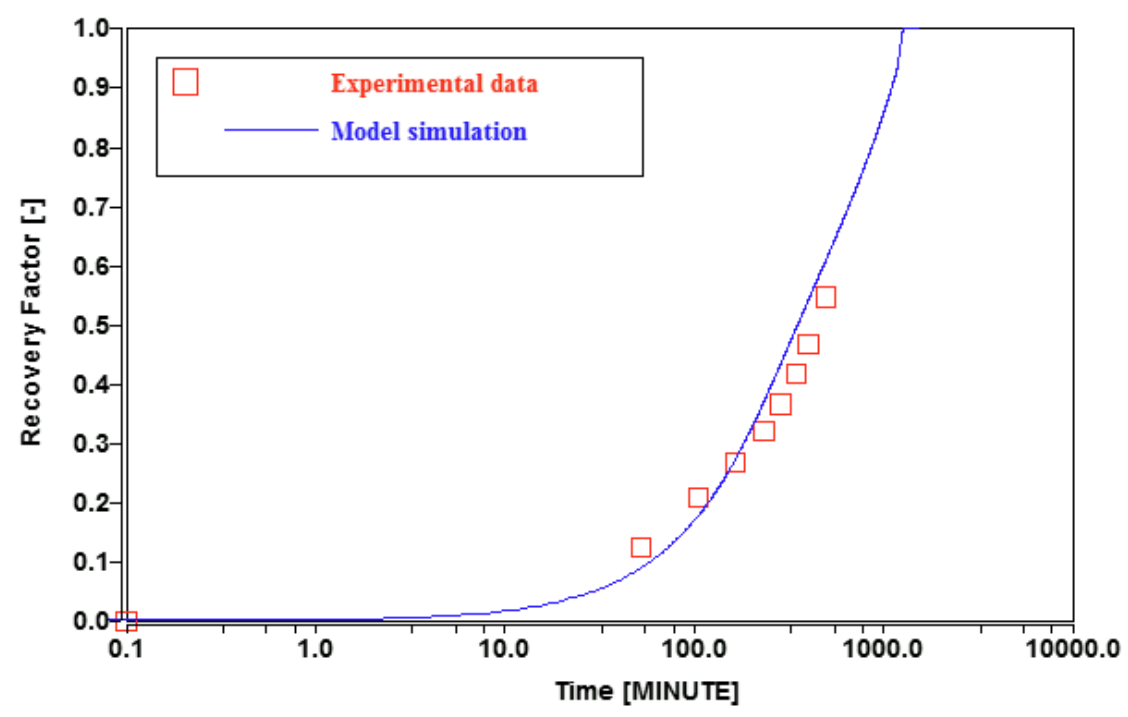

Figure 18 Recovery history obtained from simulation and experimental data for experiment1. 


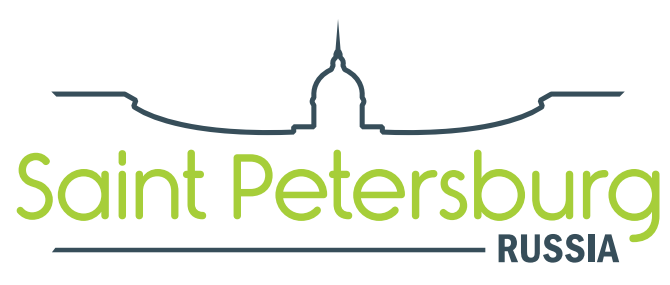

4.2. Simulation of Experiment 2: Figure 19 compares the simulations results with the experimental results obtained from Experiment 2. The result is shown in Figure 19 as blue dashed line. It can be observed that the applied model is able to reproduce the experimental data adequately. The simulation was also performed for the case that diffusion is also implemented in the model. The diffusion coefficient for hexane-hexadecane system was obtained from Bidlack and Anderson (1964) and Wesselingh and Bollen (1997) and assumes a value of $0.866 \times 10^{-9} \mathrm{~m}^{2} / \mathrm{s}$. The result is shown in Figure 19 as the dark dashed line. According to Figure 19, including molecular diffusion in the model does not lead to a significant difference between two cases considered. This means, for the system under consideration, gravity-driven forces are the main controlling mechanism and production rate is not dependent on the diffusion process. However, if only diffusion is considered (brown curve in Figure 19), the model deviates from experimental data and the recovery profile is much lower than the experimental data. This shows that the displacement process is mainly governed by gravity forces.

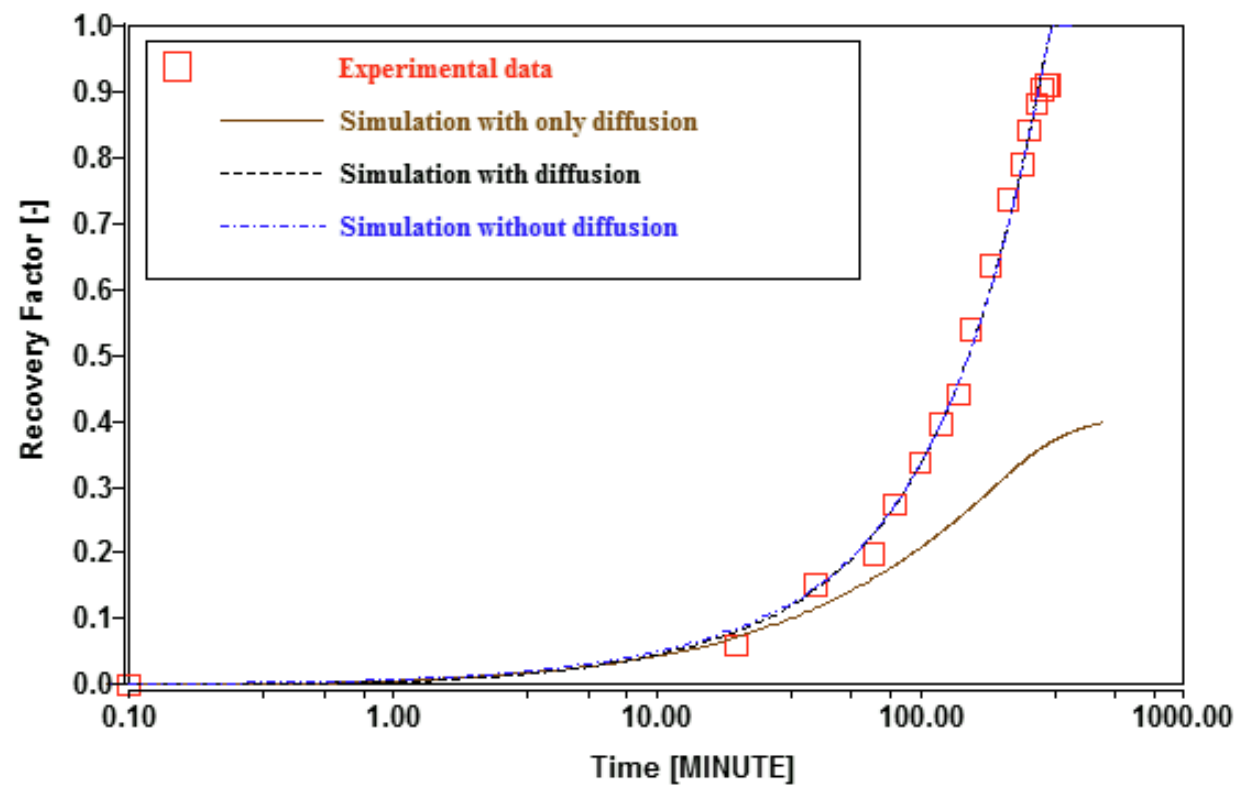

Figure 19 Recovery history obtained from simulation and experimental data for experiment 2. Three scenarios are examined. Without diffusion, with diffusion, and only diffusion.

Figure 20 shows the experimental solvent (i.e., hexane) concentration contours inside the core at different times. As it can be observed from Figure 20, the concentration profiles are basically horizontal except for the lower part of core. The concentration of hexane increases gradually from the top of the core. This observation is consistent with the idea that the displacement process in the hexadecane-hexane system is mainly governed by gravitational forces. This idea can be further confirmed by plotting the simulated average concentration profiles of hexane along the core at different times as shown in Figure 21. Comparing Figure 20 and Figure 21 reveal that the model reproduces the experimentally observed concentration profiles reasonably well. A concentration map of hexadecane at 100 minutes simulation time is depicted in Figure 22. The solvent is shown in red and oil in matrix is shown in light blue. This concentration map is comparable to that saturation profile obtained experimentally (see Figure 20). Therefore, it can be concluded that the applied model is able to capture the physics of the process and may be used to investigate the effect of operating parameters on the performance of miscible solvent injection in NFRs. 

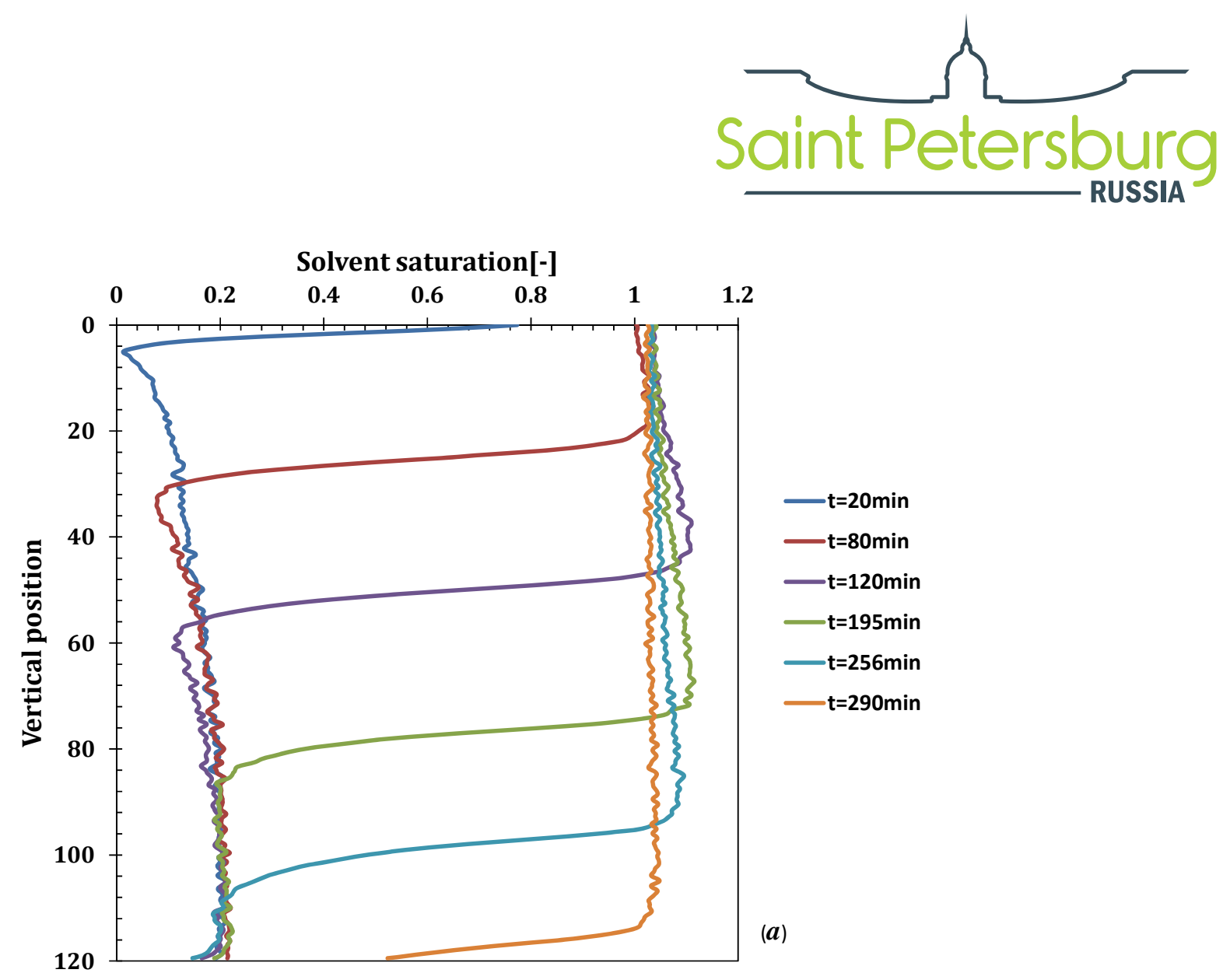

Figure 20 Concentration profiles of hexane (Experiment 1) along the core at different times. The horizontal profiles show that the solvent invades the core from the top and process is governed by gravity force.

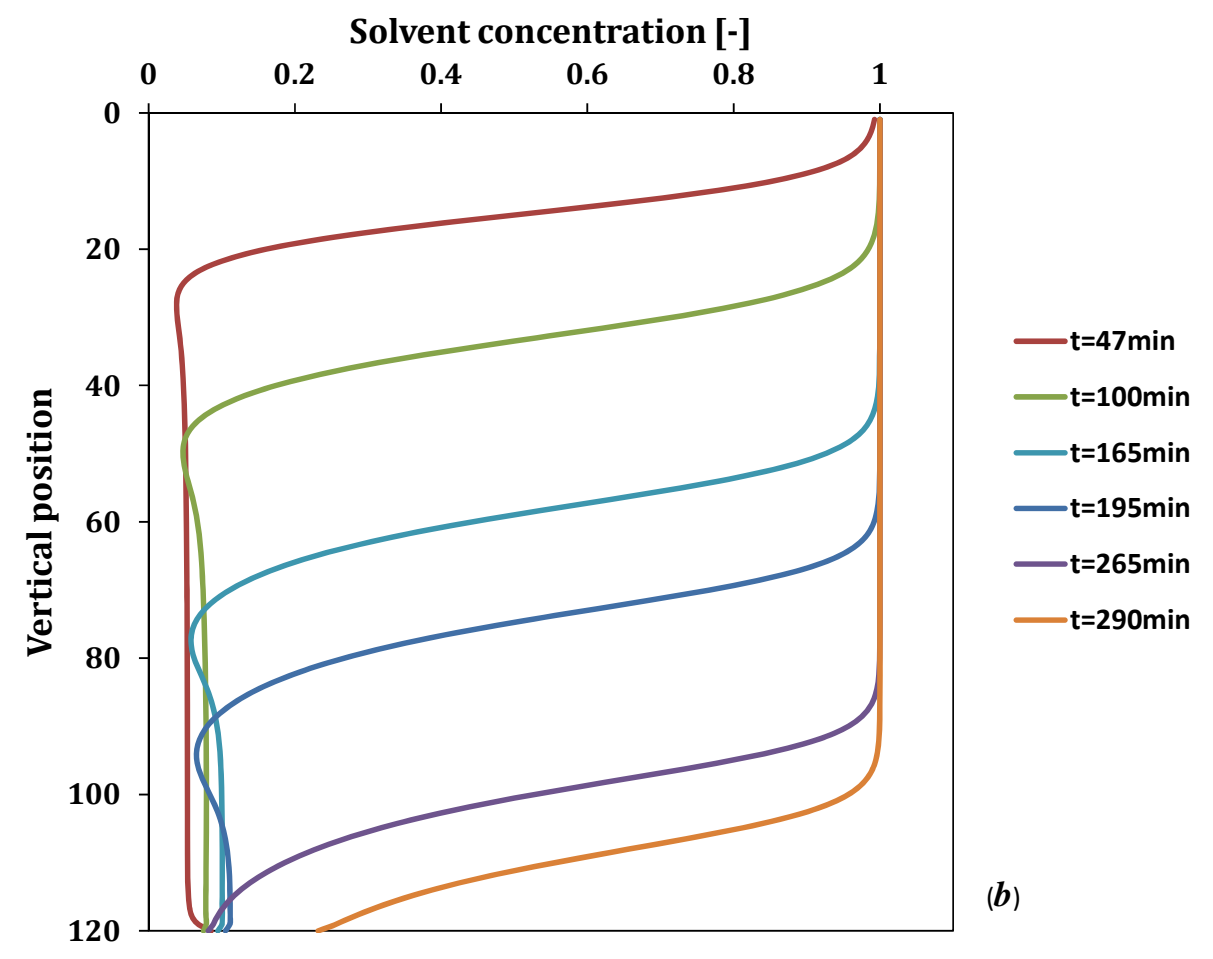

Figure 21 Simulation results for the concentration profiles of hexane (Experiment 1) along the core at different times. The horizontal profiles show that the solvent invades the core mainly from the top and process is governed by gravity force, cf. Fig. 19. 
Figure 22 Concentration map of hexadecane-hexane system (Experiment 1) obtained from simulation. Solvent invades the core mainly from the top and to a lesser extent from the lower part of the core. The process id governed by gravity forces.

4.3. Simulation of Experiment 5: Figure 23 shows the production history of Experiment 5 obtained from the simulation and the experiment. The model without diffusion (dashed line in Figure 23) gives lower production rates than the experimental data at early times of the experiment. Disregarding the diffusion process leads to an underestimate of the production rate in the simulation due to the high viscosity of the oil. When a realistic liquid-liquid diffusion coefficient is included, i.e., $0.5 \times 10^{-9} \mathrm{~m}^{2} / \mathrm{s}$, the simulation model is able to reproduce the experimental data reasonably well. The diffusion coefficient was adjusted as a fitting parameter so that the model can match the experimental data.

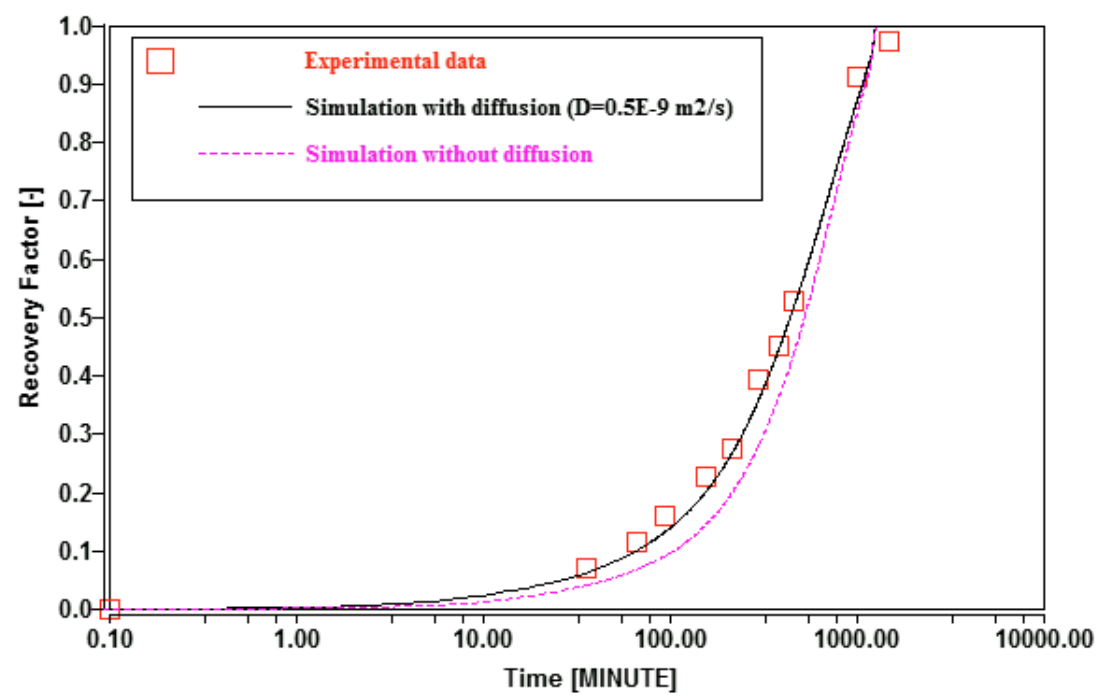

Figure 23 Recovery history obtained from simulation and experimental data for experiment 5. Two scenarios are examined; without diffusion and with diffusion. In the case of simulation without diffusion, the simulation results in lower production rates than observed in the experiment. The simulation with a realistic liquid-liquid diffusion coefficient reproduces the experimental data reasonably well. 


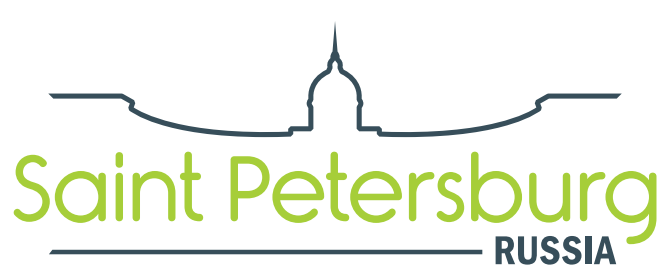

4.4. Simulation of Experiment 7: The comparison between the experimental and simulation results for Experiment 7 is shown in Figure 24. According to Figure 24, the model can match the experimental data within an acceptable range of accuracy when diffusion is included. Here the diffusion coefficient was adjusted as a fitting parameter to reproduce the experimental data and minimize the mismatch between model and experiment. Note that a low permeable core (i.e., $250 \mathrm{mD}$ ) was used in Experiment 7 as porous medium. Also, the model oil, viz. Squalane, is relatively viscous. Therefore, it is expected that diffusion plays a role in mixing between solvent and oil and should be included in the model. The model simulation without diffusion underestimates the production rate and leads to a recovery curve lower than the experimental data.

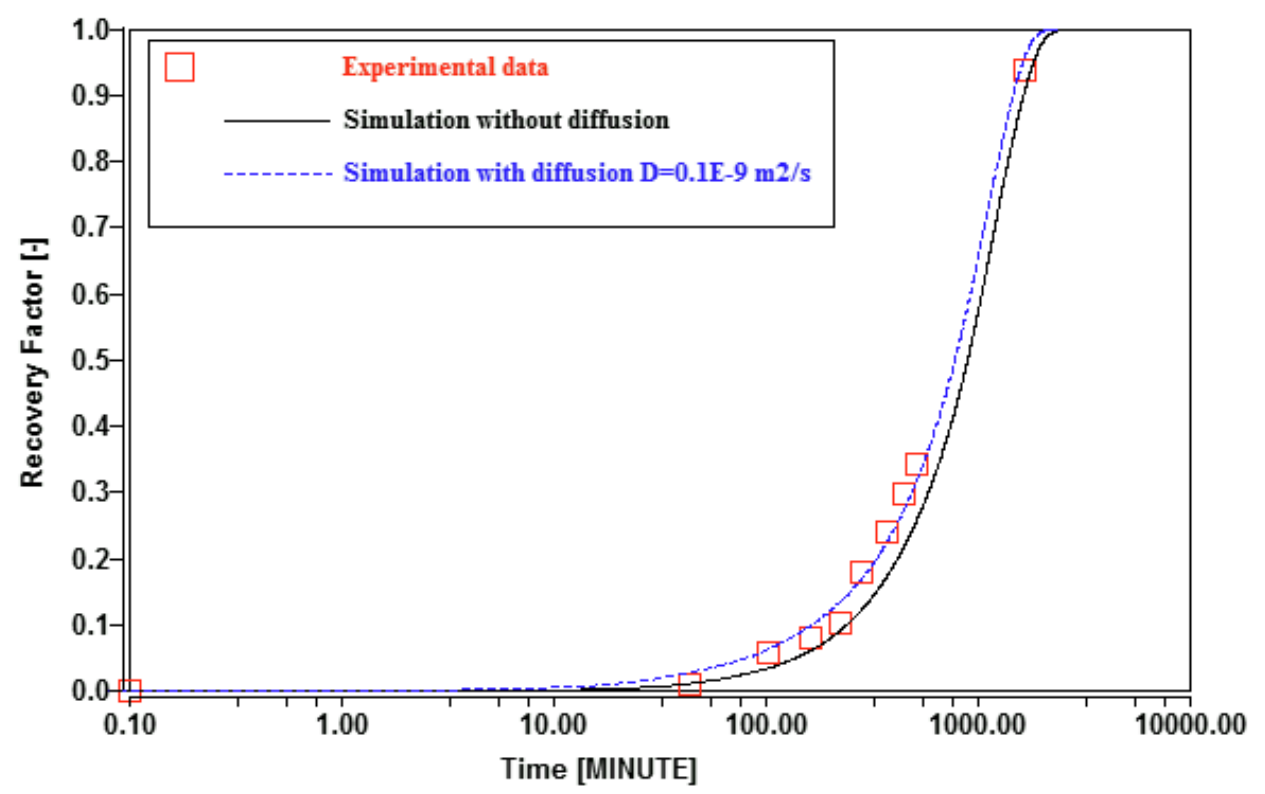

Figure 24 Recovery profile obtained from experiment and simulation for experiment 7. In the case of simulation without diffusion, the simulation results in lower production rates than the experiment. The simulation with a realistic liquid-liquid diffusion coefficient reproduces the experimental data reasonably well.

\section{Conclusions}

- It is possible to compare experimental results of oil recovery by solvents in fractured systems, with simulation data. The experiments use CT-scan data and overall mass balance measurements.

- The behavior depends on the properties of the oil and solvent, permeability of the matrix blocks and the injection flow rate.

- For faster recovery (i.e., higher drainage rates) the process should be performed at higher injection rates. This costs more solvent. However, if the amount of solvent injected is the critical parameter, the process should be preferably performed at lower flow rates.

- For the conditions that the fracture system is kept fresh with the solvent (higher injection rates), there is a potential production rate, above which the recovery history is no longer influenced by the injection rate.

- The dependence on the oil viscosity is relatively small for the conditions considered in this study. This indicates that solvent recovery can be effective for medium viscosity oils.

- The applied model can reproduce the recovery histories and saturation profiles reasonably well. For a higher oil viscosity and a lower core permeability, realistic liquid-liquid diffusion coefficients were used to minimize the mismatch between the experimentally obtained data and simulation results. 


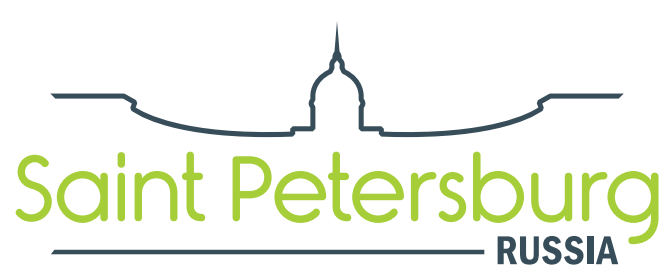

\section{Acknowledgment}

The authors gratefully acknowledge experimental support from Ellen Meijvogel-de Koning. This research was carried out within the context of the Recovery Factory program, a joint project of Shell Global Solutions International and Delft University of Technology. We gratefully acknowledge Shell Global Solutions International for the permission to publish this work.

\section{Appendix}

The viscosity $\mu_{\text {mix }}$ and density $\rho_{\text {mix }}$ of the mixture were calculated as:

$$
\begin{gathered}
\mu_{\text {mix. }}=\mu_{\text {oil }}^{x_{o l l}} \cdot \mu_{\text {solve. }}^{x_{\text {solv }}} \\
\rho_{\text {mix. }}^{-1}=\frac{w_{o i l}}{\rho_{\text {oil }}}+\frac{w_{\text {solv. }}}{\rho_{\text {solv. }}}
\end{gathered}
$$

where $x_{i}$ is mole fraction of component $i$ and $w_{i}$ is mass fraction of component $i$ in the mixture.

\section{References}

Berg, S., Oedai, S., Landman, A. J., Brussee, N., Boele, M., Valdez, R. and Gelder, K. v. [2010] Miscible displacement of oils by carbon disulfide in porous media: Experiments and analysis. Physics of Fluids 22(11), 113102.

Bidlack, D. L. and Anderson, D. K. [1964] Mutual Diffusion in the Liquid System HexaneHexadecane. The Journal of Physical Chemistry 68(1), 206-208.

Er, V. and Babadagli, T. [2010] Miscible Interaction Between Matrix and Fracture: A Visualization and Simulation Study. SPE Reservoir Evaluation \& Engineering 13(1), pp. 109-117.

Farajzadeh, R., Andrianov, A. and Zitha, P. L. J. [2009] Investigation of Immiscible and Miscible Foam for Enhancing Oil Recovery. Industrial \& Engineering Chemistry Research 49(4), 1910-1919.

Farajzadeh, R., Muruganathan, R. M., Rossen, W. R. and Krastev, R. [2011] Effect of gas type on foam film permeability and its implications for foam flow in porous media. Advances in Colloid and Interface Science 168(1-2), 71-78.

Firoozabadi, A. [2000] Recovery Mechanisms in Fractured Reservoirs and Field Performance. Journal of Canadian Petroleum Technology 39(11), 13-17.

Firoozabadi, A. and Markeset, T. I. [1994] Miscible Displacement in Fractured Porous Media: Part I-Experiments. SPE/DOE Improved Oil Recovery Symposium, Tulsa, Oklahoma.

Hatiboglu, C. U. and Babadagli, T. [2008] Diffusion mass transfer in miscible oil recovery: Visual experiments and simulation. Transport in Porous Media 74(2), 169-184.

Hoteit, H. and Firoozabadi, A. [2009] Numerical Modeling of Diffusion in Fractured Media for GasInjection and -Recycling Schemes. SPE Journal 14(2).

Jamshidnezhad, M., Montazer-Rahmati, M. and Sajjadian, V. [2004] Theoretical and Experimental Investigations of Miscible Displacement in Fractured Porous Media. Transport in Porous Media 57(1), 59-73. 


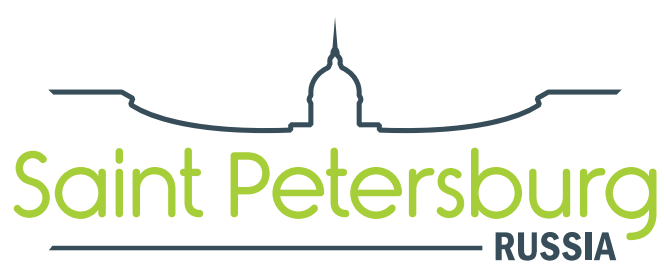

Kahrobaei, S., Farajzadeh, R., Suicmez, V. S. and Bruining, J. [2012] Gravity-Enhanced Transfer between Fracture and Matrix in Solvent-Based Enhanced Oil Recovery. Industrial \& Engineering Chemistry Research 51(44), 14555-14565.

Salimi, H. and Bruining, J. [2010] Improved Prediction of Oil Recovery From Waterflooded Fractured Reservoirs Using Homogenization. SPE Reservoir Evaluation \& Engineering 13(1), 44-55.

Simjoo, M., Dong, Y., Andrianov, A., Talanana, M. and Zitha, P. L. J. [Year] A CT scan study of immiscible foam flow in porous media for EOR, SPE 155633. SPE EOR Conference at Oil and Gas West Asia, 859-877.

Simjoo, M., Nguyen, Q. P. and Zitha, P. L. J. [2011] Rheological Transition during Foam Flow in Porous Media. SPE/DGS Saudi Arabia Section Technical Symposium and Exhibition. Society of Petroleum Engineers, Al-Khobar, Saudi Arabia.

Tan, C. T. and Firoozabadi, A. [1995] Theoretical Analysis of Miscible Displacenllent In Fractured Porous Media By a One-dinneIllsional Model: Part II - Features. 34(2).

Trivedi, J. and Babadagli, T. [2008] Efficiency of diffusion controlled miscible displacement in fractured porous media. Transport in Porous Media 71(3), 379-394.

Uleberg, K. and Høier, L. [2002] Miscible Gas Injection in Fractured Reservoirs, SPE 75136. SPE/DOE Improved Oil Recovery Symposium, Tulsa, Oklahoma.

Verlaan, M. and Boerrigter, P. M. [2006] Miscible Gas-Oil Gravity Drainage, SPE 103990. First International Oil Conference and Exhibition in Mexico, Cancun, Mexico.

Wesselingh, J. A. and Bollen, A. M. [1997] Multicomponent Diffusivities from the Free Volume Theory. Chemical Engineering Research and Design 75(6), 590-602.

Yanze, Y. and Clemens, T. [2012] The Role of Diffusion for Nonequilibrium Gas Injection Into a Fractured Reservoir. SPE Reservoir Evaluation \& Engineering 15(1), 60-71. 